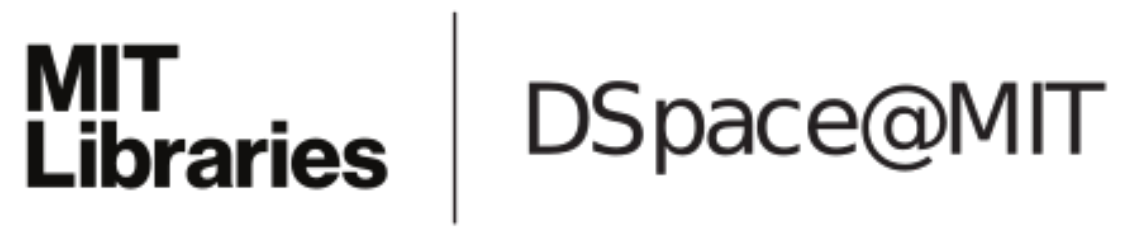

\author{
MIT Open Access Articles
}

\section{A NEW DYNAMICAL MODEL FOR THE BLACK HOLE BINARY LMC X-1}

The MIT Faculty has made this article openly available. Please share how this access benefits you. Your story matters.

Citation: Orosz, Jerome A., Danny Steeghs, Jeffrey E. McClintock, Manuel A. P. Torres, Ivan Bochkov, Lijun Gou, Ramesh Narayan, et al. "A NEW DYNAMICAL MODEL FOR THE BLACK HOLE BINARY LMC X-1." The Astrophysical Journal 697, no. 1 (May 4, 2009): 573-591. (C) 2009 American Astronomical Society.

As Published: http://dx.doi.org/10.1088/0004-637x/697/1/573

Publisher: Institute of Physics/American Astronomical Society

Persistent URL: http://hdl.handle.net/1721.1/96090

Version: Final published version: final published article, as it appeared in a journal, conference proceedings, or other formally published context

Terms of Use: Article is made available in accordance with the publisher's policy and may be subject to US copyright law. Please refer to the publisher's site for terms of use. 


\section{A NEW DYNAMICAL MODEL FOR THE BLACK HOLE BINARY LMC X-1*}

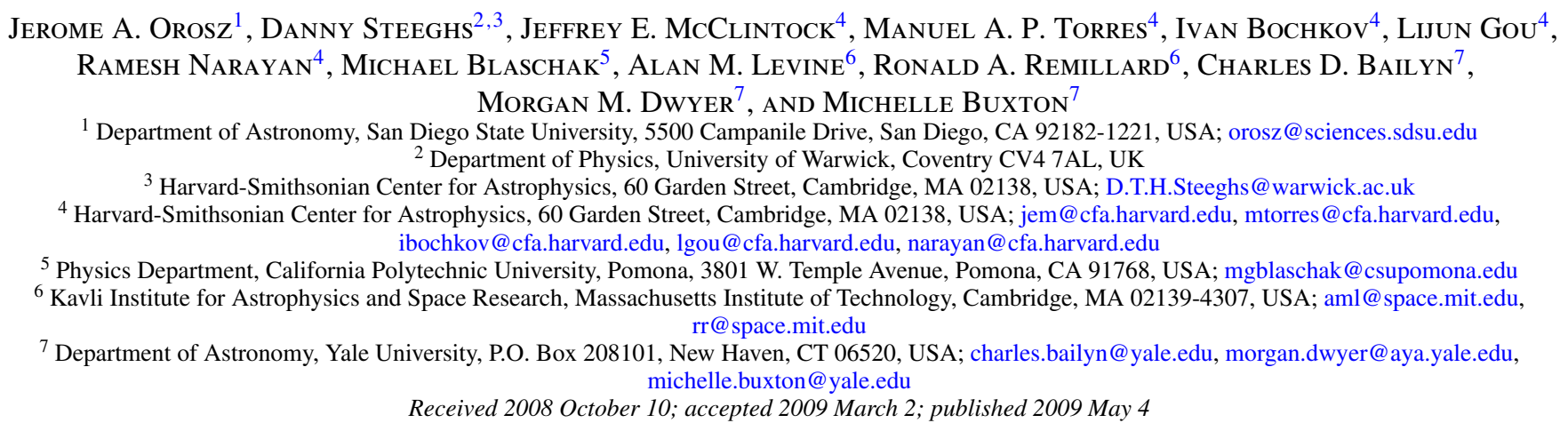

\section{ABSTRACT}

We present a dynamical model of the high mass X-ray binary LMC X-1 based on high-resolution optical spectroscopy and extensive optical and near-infrared photometry. From our new optical data we find an orbital period of $P=3.90917 \pm 0.00005$ days. We present a refined analysis of the All Sky Monitor data from $R X T E$ and find an $\mathrm{X}$-ray period of $P=3.9094 \pm 0.0008$ days, which is consistent with the optical period. A simple model of Thomson scattering in the stellar wind can account for the modulation seen in the X-ray light curves. The $V-K$ color of the star $(1.17 \pm 0.05)$ implies $A_{V}=2.28 \pm 0.06$, which is much larger than previously assumed. For the secondary star, we measure a radius of $R_{2}=17.0 \pm 0.8 R_{\odot}$ and a projected rotational velocity of $V_{\text {rot }} \sin i=129.9 \pm 2.2 \mathrm{~km} \mathrm{~s}^{-1}$. Using these measured properties to constrain the dynamical model, we find an inclination of $i=36.38 \pm 1$.92, a secondary star mass of $M_{2}=31.79 \pm 3.48 M_{\odot}$, and a black hole mass of $10.91 \pm 1.41 M_{\odot}$. The present location of the secondary star in a temperature-luminosity diagram is consistent with that of a star with an initial mass of $35 M_{\odot}$ that is 5 Myr past the zero-age main sequence. The star nearly fills its Roche lobe $(\approx 90 \%$ or more), and owing to the rapid change in radius with time in its present evolutionary state, it will encounter its Roche lobe and begin rapid and possibly unstable mass transfer on a timescale of a few hundred thousand years.

Key words: binaries: spectroscopic - black hole physics - stars: early-type - X-rays: binaries - X-rays: individual (LMC X-1)

Online-only material: color figure

\section{INTRODUCTION}

The first X-ray source to be discovered in the Magellanic Clouds, LMC X-1 (Mark et al. 1969), is a persistently luminous $\left(L_{\mathrm{x}}>10^{38} \mathrm{erg} \mathrm{s}^{-1}\right) \mathrm{X}$-ray binary that has been observed by nearly all X-ray missions during the past 37 years (e.g., Leong et al. 1971; Cui et al. 2002). Spectroscopic studies of its optical counterpart revealed an orbital period of $\approx 4$ days and a probable mass for the compact star "near $M \approx 6 M_{\odot}$ " (Hutchings et al. 1983, 1987), making it the fourth dynamical black hole candidate to be established.

The candidacy of this black hole has had a checkered history. Even the most fundamental property of this binary system-its orbital period-was established only very recently. For nearly 20 years, the accepted orbital period was 4.2288 days, the value adopted in the dynamical study of Hutchings et al. (1987). The correct orbital period, $P=3.9081 \pm 0.0015$ days, was determined by Levine \& Corbet (2006) using RXTE All-Sky Monitor (ASM) X-ray data. The optical results reported in this paper and further analysis of the ASM data amply confirm that the Levine \& Corbet (2006) period is correct. As an interesting side note, the correct period is noted in the earlier

\footnotetext{
* Based on observations made with the Magellan $6.5 \mathrm{~m}$ Clay telescope at Las Campanas Observatory of the Carnegie Institution.
}

Hutchings et al. (1983) work as one of several candidate orbital periods.

Even the identity of the optical counterpart was not firmly established until fairly recently. Initially, based on a rather uncertain X-ray position, a B5 supergiant known as R148 was favored over what is now known to be the counterpart, an O7/O8 giant identified as "star 32" by Cowley et al. (1978). The counterpart was finally established through an analysis of multiple ROSAT HRI observations by Cowley et al. (1995). A precise Chandra X-ray position (Cui et al. 2002) and the agreement between the X-ray and optical periods (mentioned above) leave no doubt that star 32 of Cowley et al. is the counterpart of LMC X-1. We present high-resolution $V$ - and $K$-band finding charts of the field in Figure 1.

In this paper, we confirm the basic model and the principal conclusions presented by Hutchings et al. (1987) while greatly improving upon their pioneering work. Thirty high-resolution and 14 medium-resolution spectra have allowed us to reduce the uncertainty in the radial velocity amplitude $K$ by a factor of 6 and to obtain a secure value for the rotational line broadening. Of equal importance, we present the first optical light curves and infrared magnitudes and colors of LMC X-1, the analysis of which allows us to strongly constrain the orbital inclination angle and hence our dynamical model of the binary. In Sections 2 and 3 we present our new photometric and spectroscopic 


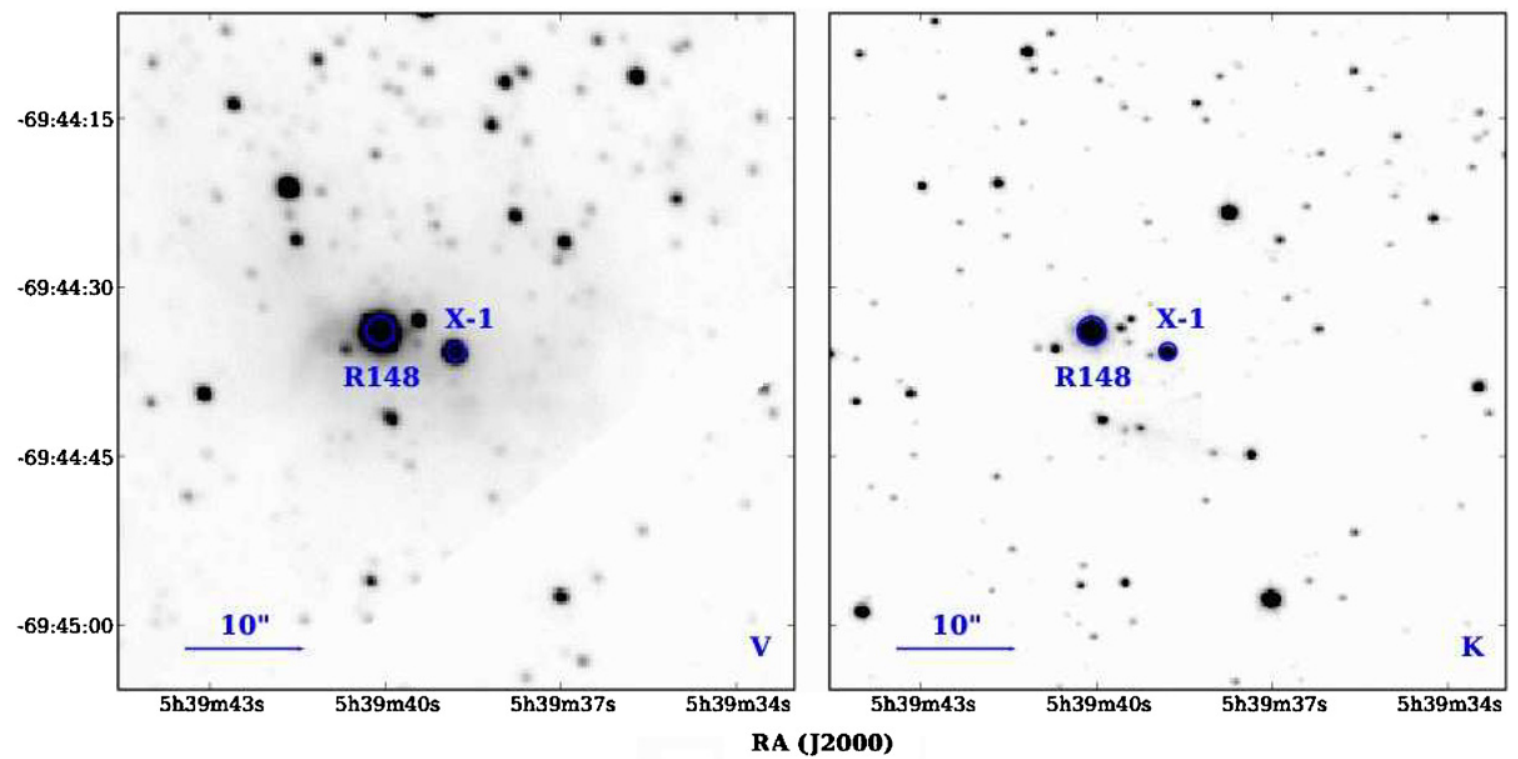

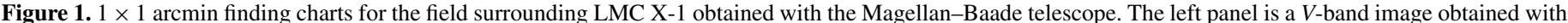

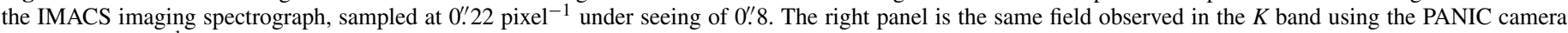
with $0{ }^{\prime} .125$ pixel $^{-1}$ sampling and a seeing of 0.55 . The correct counterpart together with the nearby B5 supergiant R148 are marked.

(A color version of this figure is available in the online journal.)

observations. In Section 4 we present improved measurements of the orbital period, the temperature of the secondary star, and the radius of the secondary star. These new observations are used to construct a dynamical model of the system, which is presented in Section 5; a summary of our results is in Section 6.

\section{PHOTOMETRY}

\subsection{Observations and Reductions}

Optical and near infrared observations of LMC X-1 were obtained on 66 nights between 2007 January 6 and 2007 April 2 using the ANDICAM $^{8}$ instrument on the $1.3 \mathrm{~m}$ telescope at CTIO, which is operated by the Small and Moderate Aperture Research Telescope System (SMARTS) consortium. ${ }^{9}$ During each visit to the source, a $120 \mathrm{~s}$ exposure in the $B$ filter and a $120 \mathrm{~s}$ exposure in the $V$ filter were obtained simultaneously with an exposure in $J$ that consisted of 10 dithered subexposures of $12 \mathrm{~s}$ each. Flat field images for all filters were taken nightly, and IRAF tasks were used to perform the standard image reductions (i.e., bias subtraction and flat-fielding for the optical and flat-fielding, sky subtraction, and image combination for the infrared). All of the images were inspected visually, and a few of them were discarded owing to poor signal-to-noise. We retained 58 images in $B, 57$ images in $V$, and 57 images in $J$.

We also observed the field of LMC X-1 on the nights of 2006 December 6 and December 8 using the 6.5 m MagellanBaade telescope at Las Campanas Observatory (LCO) and the Persson's Auxiliary Nasmyth Infrared Camera (PANIC; Martini et al. 2004). On December 6 three composite frames were obtained in the $J$ band and four composite frames were obtained in the $K$ band in 0.7 seeing. Each composite frame consists of five $7 \mathrm{~s}$ dithered subexposures. On December 8, 10 sequences in $J$ with $3 \mathrm{~s}$ subexposures and eight sequences in $K$ with $7 \mathrm{~s}$ subexposures were obtained in 0'.5 seeing. The conditions were photometric on both nights. The PANIC data were reduced and

\footnotetext{
8 See http://www.astronomy.ohio-state.edu/ANDICAM.

9 See http://www.astro.yale.edu/smarts
}

processed with IRAF and custom PANIC software to produce mosaic frames of the field (13 mosaic frames in $J$ and 12 mosaic frames in $K$ ).

\subsection{Derivation of the Photometric Light Curves}

PSF-fitting photometry was used on the PANIC mosaic images to obtain instrumental magnitudes of LMC X-1 and two nearby stars. The absolute calibration was done with respect to stars from the Two Micron All Sky Survey (2MASS) catalog (Skrutskie et al. 2006). Comparison stars having less than 8000 detector counts in the PANIC frames were selected and checked for variability. Weighted differential photometry of LMC X-1 was performed with respect to seven and six comparison stars for the $J$ - and $K$-band mosaic images, respectively. For LMC $\mathrm{X}-1$, we found a mean $J$ magnitude of $J=13.76$ with an $\mathrm{rms}$ of 0.02 mag and a mean $K$ magnitude of $K=13.43$ with an rms of $0.01 \mathrm{mag}$. Optimal aperture photometry was also performed for LMC X-1 and the comparison stars, and we obtained similar values for the mean magnitudes. For comparison, the magnitudes for LMC X-1 given in the 2MASS catalog are $J=13.695 \pm 0.063$ and $K=13.293 \pm 0.063$, although these values should be treated with caution owing to the presence of the bright star R148 $\approx 6^{\prime \prime}$ to the east (Figure 1).

The photometric time series for the $B$ and $V$ filters were obtained using the programs DAOPHOT IIe, ALLSTAR, and DAOMASTER (Stetson 1987, 1992a, 1992b; Stetson et al. 1991). The instrumental magnitudes were placed on the standard scales using observations of the Landolt (1992) fields RU 149, PG 1047+003, and PG 1657+078. Aperture photometry was done on the standard stars and on several bright and isolated stars in the LMC X-1 field and the DAOGROW algorithm (Stetson 1990) was used to obtain optimal magnitudes. We find for LMC $\mathrm{X}-1$ an average $V$ magnitude of $V=14.60 \pm 0.02$, and a $B-V$ color of $B-V=0.17 \pm 0.08$, marginally consistent with the values of $V=14.52 \pm 0.05$ and $B-V=0.29 \pm 0.02$ given in Bianchi \& Pakull (1985).

The photometric time series for the SMARTS $J$ band was obtained using aperture photometry. A relatively small aperture 


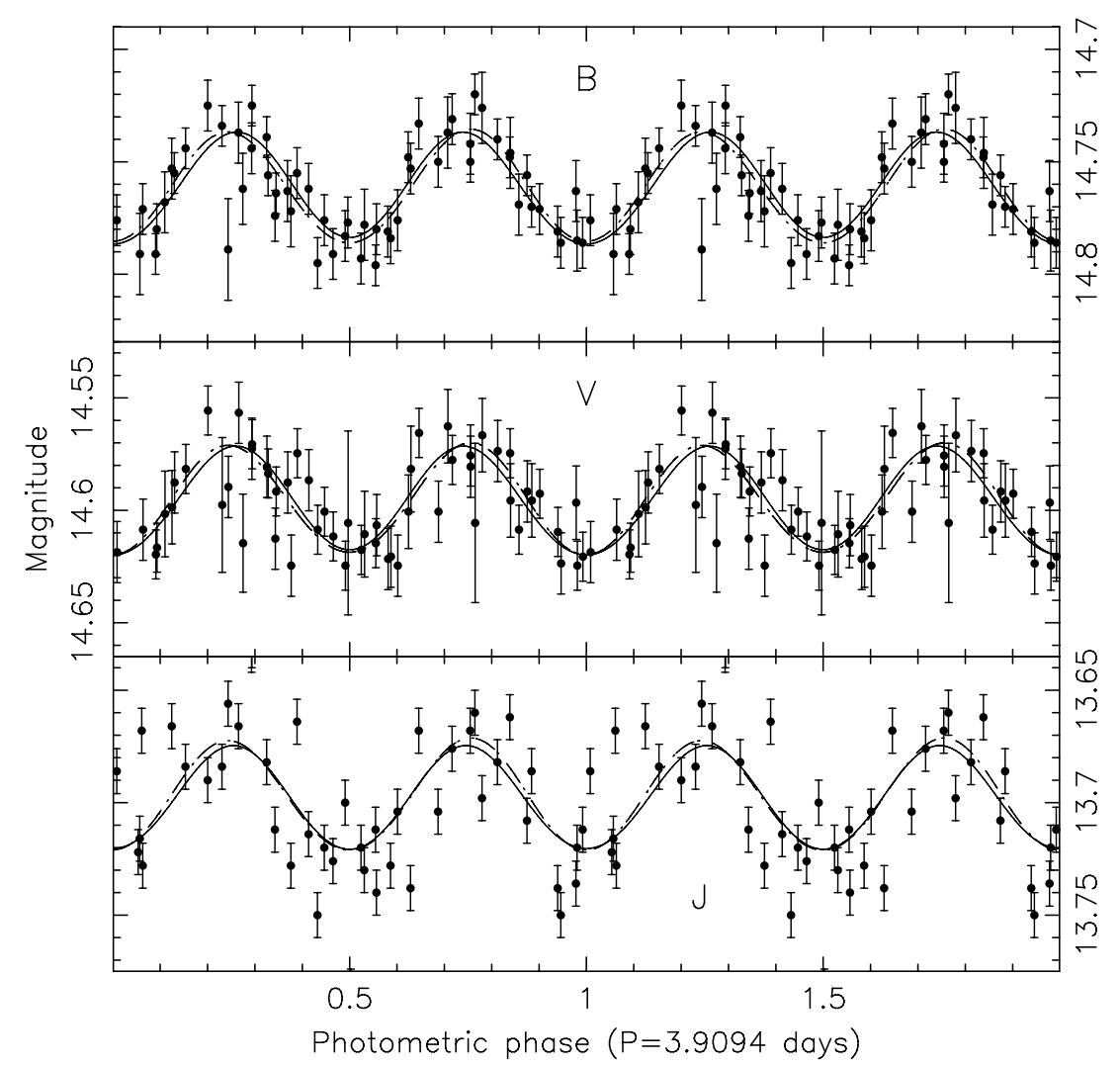

Figure 2. Phased light curves of LMC X-1. Phase zero corresponds to the time of the inferior conjunction of the secondary star. Shown from the top are the $B, V, J$ light curves with the best-fitting ellipsoidal models assuming a circular orbit (solid lines) and assuming an eccentric orbit (dashed lines). Owing to the large scatter, the $J$-band data were not used in the modeling.

radius $\left(\approx 1^{\prime \prime}\right)$ was used to exclude light from nearby sources. Stars from the 2MASS catalog were used to place the instrumental magnitudes onto the standard system. The $J$-band magnitudes from SMARTS are in agreement with the mean $J$-band magnitude derived from the PANIC data.

Figure 2 shows the light curves phased on the photometric ephemeris determined below. The light curves show the doublewave modulation characteristic of ellipsoidal variations, with maxima at the quadrature phases and minima at the conjunction phases. The amplitude of the modulation $(\approx 0.06 \mathrm{mag}$, maximum to minimum) is not especially large, which usually indicates a relatively low inclination, or a small Roche lobe filling factor for the mass donor, or both.

\section{SPECTROSCOPY}

\subsection{Observations and Reductions}

Thirty spectra of LMC X-1 were obtained on the nights of 2005 January 19-24 using the Magellan Inamori Kyocera Echelle (MIKE) spectrograph (Bernstein et al. 2002) and the $6.5 \mathrm{~m}$ Magellan Clay telescope at LCO. The instrument was used in the standard dual-beam mode with a $1^{\prime \prime} .0 \times 5^{\prime \prime} 0$ slit. Exposure times ranged between 1200 and $2700 \mathrm{~s}$ and observing conditions were good. The seeing was well below $1^{\prime \prime}$ most of the time with excursions as low as 0.5 and as high as $1^{\prime \prime} .2$. The pair of $2048 \times 4096$ pixel CCD detectors were operated in the $2 \times 2$ on-chip binning mode. The blue arm had a wavelength coverage of 3340-5065 $\AA$ and the spectral dispersion on the MIT detector was $\lambda / \Delta \lambda=100,000\left(0.03-0.05 \AA\right.$ pixel $\left.^{-1}\right)$, whereas the red arm covered $4855-9420 \AA$ and the dispersion on its SITe detector was $\lambda / \Delta \lambda=71,000\left(0.07-0.13 \AA\right.$ pixel $\left.^{-1}\right)$. Our 1" slit delivered a spectral resolution of $R=33,000$ and 28,000 in the blue and red arms respectively. ThAr lamp exposures were obtained before and after each pair of observations of the object, and several flux standards and spectral-comparison stars were observed.

The MIKE was located at a Nasmyth focus, which minimizes flexure and calibration problems. No instrument rotator or dispersion compensation optics were available, and the position angle of the spectrograph was set to the parallactic angle for an object at air mass 1.3. Because all of our observations were taken below air mass $\approx 1.5$, the effects of light loss due to atmospheric dispersion are small and unlikely to significantly affect our results.

The MIKE data were reduced using a pipeline written by Dan Kelson. ${ }^{10}$ The pipeline performs all of the detector calibrations in the standard way using bias frames and tungsten flat fields which were obtained each afternoon. The arc spectra taken at the position of each target were used to correct for the nonorthogonality between the dispersion and spatial axes and construct a two-dimensional wavelength solution for all object frames. All object orders were then sky subtracted using the technique discussed in Kelson (2003) and optimally extracted. The signal-to-noise ratio per pixel in the orders with useful lines was generally in the range of $\approx 50-100$ for most of the spectra.

The extracted spectra were inspected visually and artifacts due to cosmic rays were removed manually using simple interpolation. In addition to cosmic rays, there were some artifacts in the cores of the higher Balmer lines owing to

\footnotetext{
${ }_{10} \mathrm{http}: / /$ www.ociw.edu/Code.
} 


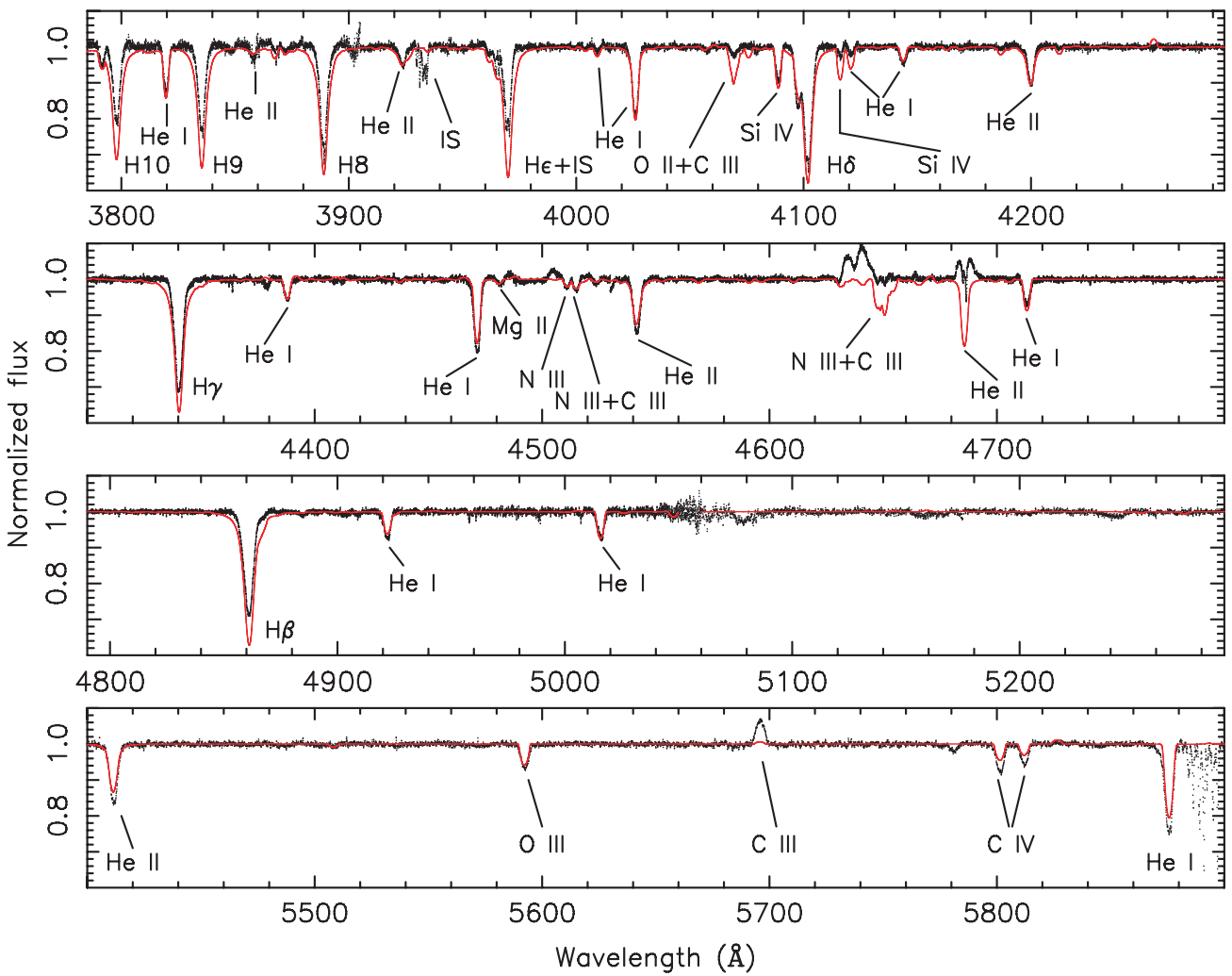

Figure 3. Average spectrum of LMC X-1 in the rest frame of the secondary star (black dots) obtained with the MIKE. Most of the strong features are labeled, and interstellar lines are marked with "IS". The best-fitting model (red line) has $T_{\text {eff }}=33,225 \mathrm{~K}, \log g=3.56$, and $V_{\text {rot }} \sin i=128.0 \mathrm{~km} \mathrm{~s}{ }^{-1}$. The noise near $5060 \AA$ is caused by the transition from the blue arm to the red arm.

imperfect subtraction of nebular lines. These artifacts were also removed by interpolation.

Finally, the individual orders in the cleaned spectra were trimmed to remove the low signal-to-noise regions at each end and normalized using cubic spline fits. The trimmed and normalized orders were then merged into a pair of spectra (e.g., red arm and blue arm). Figure 3 shows the average spectrum (in the rest frame of the secondary) for much of the blue arm and part of the red arm. The model spectrum shown in Figure 3 is discussed in Section 4.2.

An additional fourteen spectra of LMC X-1 were obtained on the nights of 2008 August 2-3 using the Magellan Echellette Spectrograph (MagE; Marshall et al. 2008) and the Clay telescope at LCO. We used the 0.7 slit which yields a resolving power of $R=6000$. The exposure times were $600 \mathrm{~s}$ and the

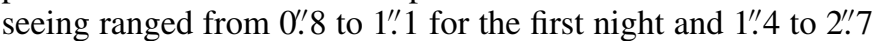
for the second night. ThAr lamp exposures were obtained before and after each pair of observations of LMC X-1.

The images were reduced with tasks in the IRAF "ccdproc" and "echelle" packages. After the bias was subtracted from each image, pairs of images were combined using a clipping algorithm to remove cosmic rays. The resulting seven images were flat-fielded using a normalized master flat and then rotated in order to align the background night sky emission lines along the columns. After the background emission lines were rectified, the spectra from individual orders were optimally subtracted. Unfortunately, the rectification of the background lines was not perfect, which produced artifacts due to nebular lines in the cores of the higher Balmer lines. These imperfections were removed by simple interpolation. The process used to merge the orders in the MIKE spectra described above was also used to merge the MagE orders. The resulting seven spectra cover a useful wavelength range of 3200-9250 .

\subsection{Radial Velocities}

In a recent study of three high mass X-ray binaries with OB-star companions, van der Meer et al. (2007) measured radial velocity curves using single lines. They found that X-ray heating produced distortions in some of the line profiles, thereby yielding for some cases different $K$-velocities for different lines.

In order to assess the effects of X-ray heating on our spectra, we selected four bandpasses with strong lines that have a wide range of excitation energies over which to derive radial velocities from the MIKE spectra: $3780-3880 \AA$, dominated by H9 \& H10 and including He I $\lambda 3820 ; 3990-4062 \AA$, He I $\lambda 4026$; 4150$4250 \AA$, He II $\lambda 4200$; and 4425-4525 ̊, He I $\lambda$ 4471. These lines were selected because of their prominence in both object and template spectra and their isolation from other spectral features.

The radial velocities were determined using the FXCOR task within IRAF, which is an implementation of the crosscorrelation analysis developed by Tonry \& Davis (1979). For each bandpass, the spectra were low-frequency filtered either by fitting a Legendre polynomial or by Fourier filtering. To optimize the removal of any order blaze remnants, the Legendre polynomial orders and the Fourier ramp filter components were determined individually for each bandpass. For example, the region containing $\mathrm{H} 9$ and $\mathrm{H} 10$ required a Legendre polynomial order of 8 (i.e., nine terms) or a Fourier cut-on wavenumber of 4 and a full-on wavenumber of 8 , whereas the region containing the line $\mathrm{He}$ I $\lambda 4471$ needed a 28 -piece polynomial or a cut-on of 9 and a full-on of 18. The spectra were then 


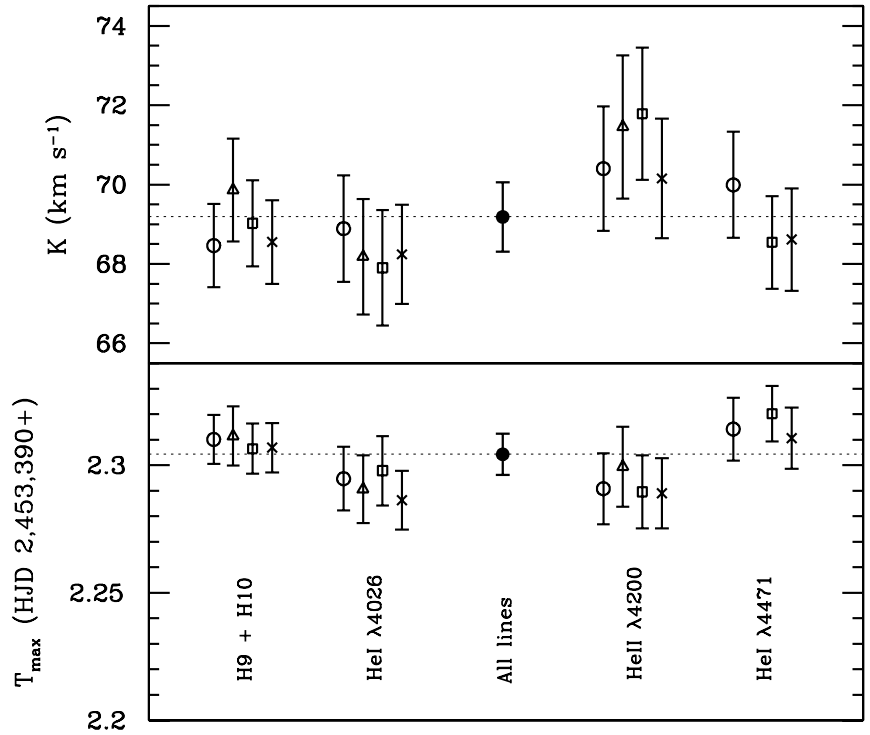

Figure 4. Summary of fitted values of the velocity semiamplitude $K$ (top) and the time of maximum velocity $T_{\max }$ (bottom) for different combinations of bandpasses, template spectra, and modes of filtering. The lines or line group are indicated at the bottom of the figure (see the text). The open and filled circles are for template HD 93843 and Legendre filtering. Triangles: HD 101205, Legendre filtering. Squares: synthetic template, Legendre filtering. Crosses: synthetic template, Fourier filtering. The filled circles and dashed lines correspond to the values of $K$ and $T_{\max }$ given in the text, which were computed for the mean velocities. The uncertainties shown are purely statistical, correspond to $\chi_{v}^{2}=1$, and are at the $1 \sigma$ level of confidence. No results are shown for the He I $\lambda 4471$ line for HD 101205 because the template spectrum was corrupted in this band.

continuum-subtracted prior to computing the cross-correlation. No other filtering was applied.

The correlations were performed using three different templates: a model spectrum from the OSTAR2002 grid (Lanz \& Hubeny 2003; see the discussion below) and the spectra of two of our comparison stars: HD 93843, O6 III(f) and HD 101205, O7 IIIn((f)) (Walborn 1972, 1973). Typical values of the Tonry \& Davis (1979) $r$-value, a measure of signal-to-noise ratio, ranged from about 10 to 70 and the median value was 26 .

The radial velocity data for each bandpass were fitted to a circular orbit model which returns the systemic velocity $V_{0}$, the time of maximum velocity $T_{\max }$, and the velocity semiamplitude of the secondary $K_{2}$. The orbital period was fixed at our adopted value, which is given in Table 3 . The model provided good fits to the data. For each fit, the statistical errors on the velocities returned by the fit were scaled by factors ranging from 0.3 to 2.1 and the data were refitted in order to give a reduced chi-squared $\left(\chi_{v}^{2}\right)$ of unity.

As an aid to visualize the systematic effects, Figure 4 summarizes the fitted values of $K_{2}$ and $T_{\max }$ obtained for different combinations of (1) the four bandpasses, (2) the three template stars, and (3) the two modes of low-frequency filtering, Legendre and Fourier. The open circles show, for example, the results obtained by fitting the velocity data using the spectrum of HD 93843 as the template and employing Legendre filtering. The other open symbols show for comparison the results obtained with the other template/filter combinations. The results shown as filled circles and bold error bars were derived using the weighted mean velocities for each spectrum: $K_{2}=69.19 \pm$ $0.88 \mathrm{~km} \mathrm{~s}^{-1}$ and $T_{\max }=\mathrm{HJD} 2453392.3043 \pm 0.0081$. These mean values are indicated by the dashed lines.

Figure 4 shows that our results for $K$ and $T_{\max }$ are quite insensitive to the choice of bandpass, template, or mode of

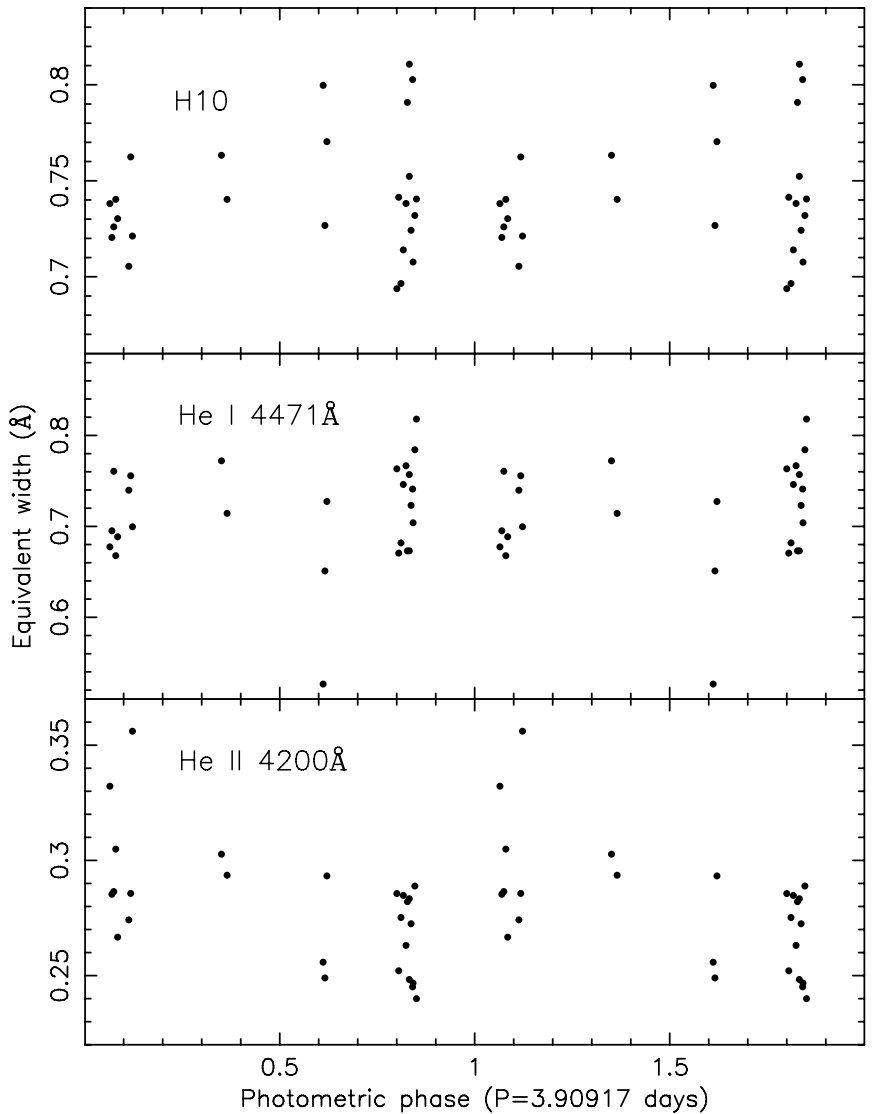

Figure 5. Equivalent widths of $\mathrm{H} 10$ (top), $\mathrm{He}$ I $\lambda 4471$ (center), and He II $\lambda 4200$ (bottom) as a function of orbital phase, where phase zero corresponds to the time of the inferior conjunction of the secondary star.

filtering, although there are some systematic effects at play. For example, the weighted mean $K$-velocity of the He II line, $K(\lambda 4200)=70.87 \pm 0.81 \mathrm{~km} \mathrm{~s}^{-1}$, is greater than our adopted mean value, albeit still within $2 \sigma$. This modest shift in $K$ is far less than was observed in the recent van der Meer et al. (2007) study of three O-star X-ray binaries that contain neutron star primaries. Because we have considered lines with a wide range of excitation energies (Balmer, $\mathrm{He}$ I and He II) and have found quite consistent results, we conclude that our dynamical results are little affected by X-ray heating, tidal distortion of the secondary, stellar wind, and other commonly observed sources of systematic effects.

Finally, we measured the equivalent widths of the H10, He I $\lambda 4471$, and He II $\lambda 4200$ lines in the individual spectra. There is no apparent trend with orbital phase (see Figure 5), which further confirms that the effects of X-ray heating are minimal.

Knowing that the velocities are little effected by X-ray heating, we derived the final adopted velocities from the MIKE and MagE spectra using FXCOR, a synthetic template, and a cross-correlation region that covers nine $\mathrm{He} \mathrm{I}$ and $\mathrm{He}$ II lines between $4000 \AA$ and $5020 \AA$. The mean velocity curve is shown plotted in Figure 6.

\section{RESULTS}

\subsection{Refined Orbital Period}

Levine \& Corbet (2006) analyzed RXTE ASM data of LMC X-1 taken between 1996 March and 2005 November. They 

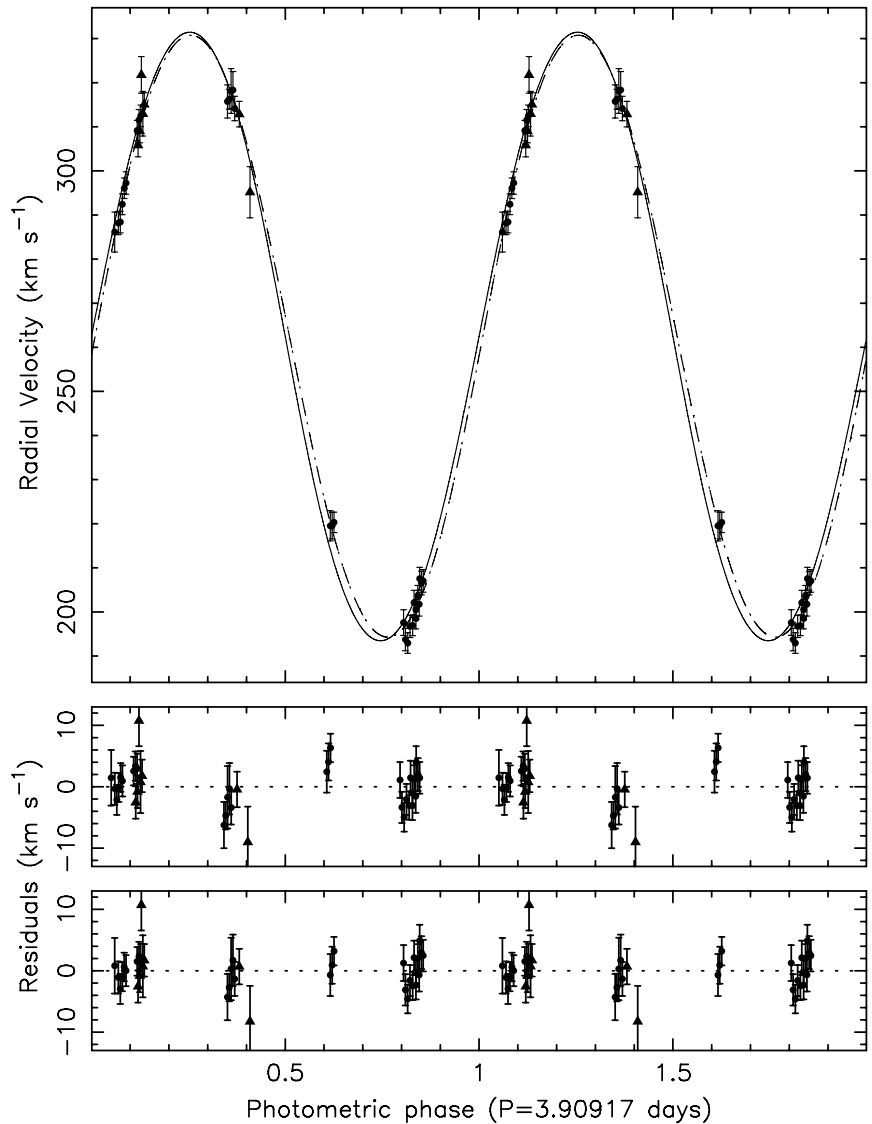

Figure 6. Top: the phased velocity curve of LMC X-1. Phase zero corresponds to the time of the inferior conjunction of the secondary star. The MIKE radial velocities are shown with the filled circles and the MagE radial velocities are shown with the filled triangles. The model curve for the best-fitting orbital model assuming a circular orbit is shown with the solid line, and the model curve for the best-fitting model assuming an eccentric orbit is shown with the dashed lines. Center: the residuals with respect to the circular orbit model. Bottom: the residuals with respect to the eccentric orbit model.

reported a periodicity of $3.9081 \pm 0.0015$ days, which differs from the period of $P=4.2288$ days given in Hutchings et al. (1987). On the other hand, the X-ray period is consistent with one of the possible periods given in Hutchings et al. (1983), namely $P=3.909 \pm 0.001$ days.

We derived a refined period from our optical data. The optical light curves completely rule out the 4.2288 day period and strongly favor the period near 3.909 days. We made a periodogram from the Magellan radial velocities by fitting a three-parameter sinusoid to the data at various trial periods and recording the $\chi^{2}$ of the fit. The results are shown in the top of Figure 7. A unique period cannot be found from these data alone, leaving us with possible periods near 3.884, 3.898, and 3.909 days. Although the radial velocities given in Hutchings et al. $(1983,1987)$ have relatively large errors and the times given are accurate to only about one minute, they can be used to rule out possible alias periods. When these additional velocities are included in the analysis, only the period near 3.909 days remains viable (see the center panel in Figure 7). Finally, we used the ELC code (Orosz \& Hauschildt 2000) to compute periodograms by modeling both our light and velocity curves together. The details of the model fitting are discussed thoroughly in Section 4.4.1. For the purposes of computing a periodogram, the period is held fixed at a given trial period and several other fitting parameters are varied (here the orbit is

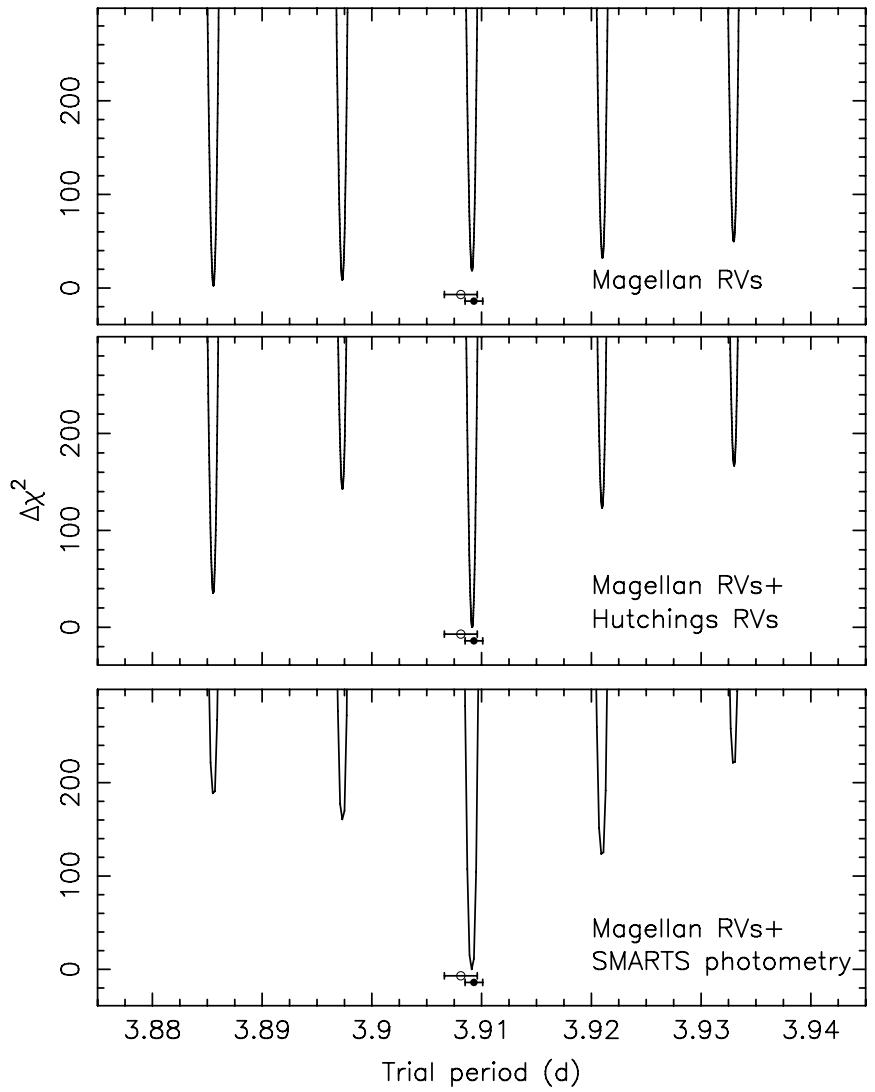

Figure 7. Top: $\chi^{2}$ computed using a three-parameter sinusoid vs. the trial period for the Magellan radial velocities. Center: the periodogram (computed in a similar manner as above) for the radial velocities from Hutchings et al. (1983, 1987) combined with the Magellan radial velocities. Bottom: the periodogram derived from the SMARTS photometry and the Magellan radial velocities (see the text). The best-fitting period is found to be $3.90917 \pm 0.00005$, and all other possible alias periods are ruled out at high confidence. In each of the three panels the X-ray period given in Levine \& Corbet (2006) is indicated with an open circle, and the refined X-ray period is denoted by the filled circle.

assumed to be circular) until the fit is optimized. The graph of $\chi^{2}$ versus $P$ (see the bottom panel of Figure 7) then serves as a periodogram. The combination of the SMARTS photometry and the Magellan radial velocities yields a unique period of $3.90917 \pm 0.00005$ days. This period is consistent with the period found from the combination of the Hutchings et al. $(1983,1987)$ and Magellan radial velocities. This period is also consistent with the X-ray period found by Levine \& Corbet (2006), and with the refined X-ray period that we now derive.

The ASM consists of three Scanning Shadow Cameras (SSCs) mounted on a rotating Drive Assembly (Levine et al. 1996). Approximately 53,700 measurements of the intensity of LMC $\mathrm{X}-1$, each from a 90 s exposure with a single SSC, were obtained from the beginning of the RXTE mission in early 1996 through 2008 June. A single exposure yields intensity estimates in each of three spectral bands which nominally correspond to photon energy ranges of $1.5-3,3-5$, and $5-12 \mathrm{keV}$ with a sensitivity of a few SSC counts s ${ }^{-1}$ (the Crab Nebula produces intensities of 27, 23 , and $25 \mathrm{SSC}$ counts $\mathrm{s}^{-1}$ in the three bands, respectively). The $\mathrm{X}$-ray intensity of LMC X-1, as seen over more than 12 years in the ASM light curves, has been more or less steady near $20 \mathrm{mCrab}(1.5-12 \mathrm{keV})$ with variations, when 10 day averages are considered, of $\approx \pm 10 \%$.

A periodicity in the X-ray intensity of LMC X-1 was found during a search for periodicities in ASM data using advanced analysis techniques. Two strategies were applied in this search to 


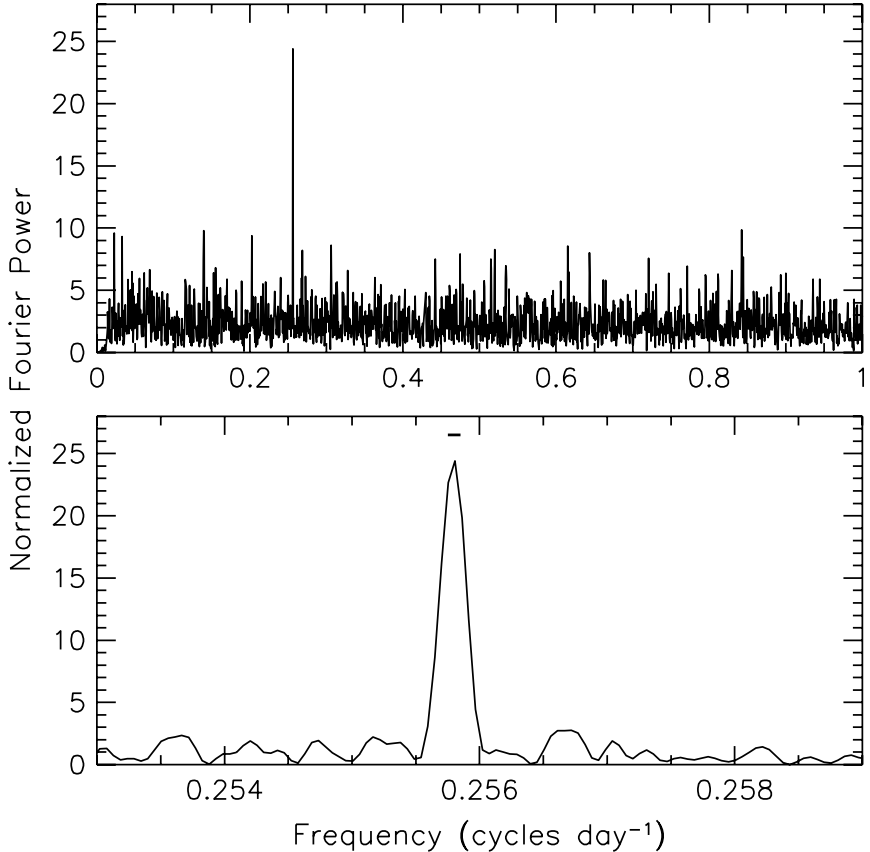

Figure 8. PDS of a $1.5-12 \mathrm{keV}$ ASM light curve of LMC X-1 that has been modified to remove variability on timescales longer than $\approx 30$ days (see the text). The original FFT was oversampled by a factor of 4 and had approximately 62,000 frequencies from 0 cycles day $^{-1}$ to the Nyquist frequency of 3.3 cycles day $^{-1}$. Top: the low frequency part of a rebinned PDS in which the number of frequency bins was reduced by a factor of 10 by using the maximum power in each contiguous set of 10 frequency bins in the original PDS as the power of the corresponding bin in the rebinned PDS. Bottom: a portion of the original PDS. The horizontal bar above the peak shows our estimate of the possible values of the centroid frequency, i.e., $0.25580 \pm 0.00005 \mathrm{day}^{-1}$. In both panels, the power is normalized relative to the PDS-wide average.

improve the sensitivity. The first involves the use of appropriate weights such as the reciprocals of the variances, since the individual ASM measurements have a wide range of associated uncertainties. The second strategy stems from the fact that the observations of the source are obtained with a low duty cycle, i.e., the window function is sparse (and complex). The properties of the window function, in combination with the presence of slow variations of the source intensity, act to hinder the detection of variations on short time scales. The power density spectrum (PDS) of the window function has substantial power at high frequencies, e.g., 1 cycle day ${ }^{-1}$ and 1 cycle per spacecraft orbit $(\approx 95$ minute period). Since the data may be regarded as the product of a (hypothetical) continuous set of source intensity measurements with the window function, a Fourier transform of the data is equivalent to the convolution of a transform of a continuous set of intensity measurements with the window function transform. The high frequency structure in the window function transform acts to spread power at low frequencies in the source intensity to high frequencies in the calculated transform (or, equivalently, the PDS). This effectively raises the noise level at high frequencies.

In our analysis, the sensitivity to high frequency variations is enhanced by subtracting a smoothed version of the light curve from the unsmoothed light curve. To perform the smoothing, we do not simply convolve a box function with the binned light-curve data since that would not yield any improvement in the noise level at high frequencies. Rather, we ignore bins which do not contain any actual measurements and we use weights based on estimates of the uncertainties in the individual measurements to compute the smoothed light curve. The kernel

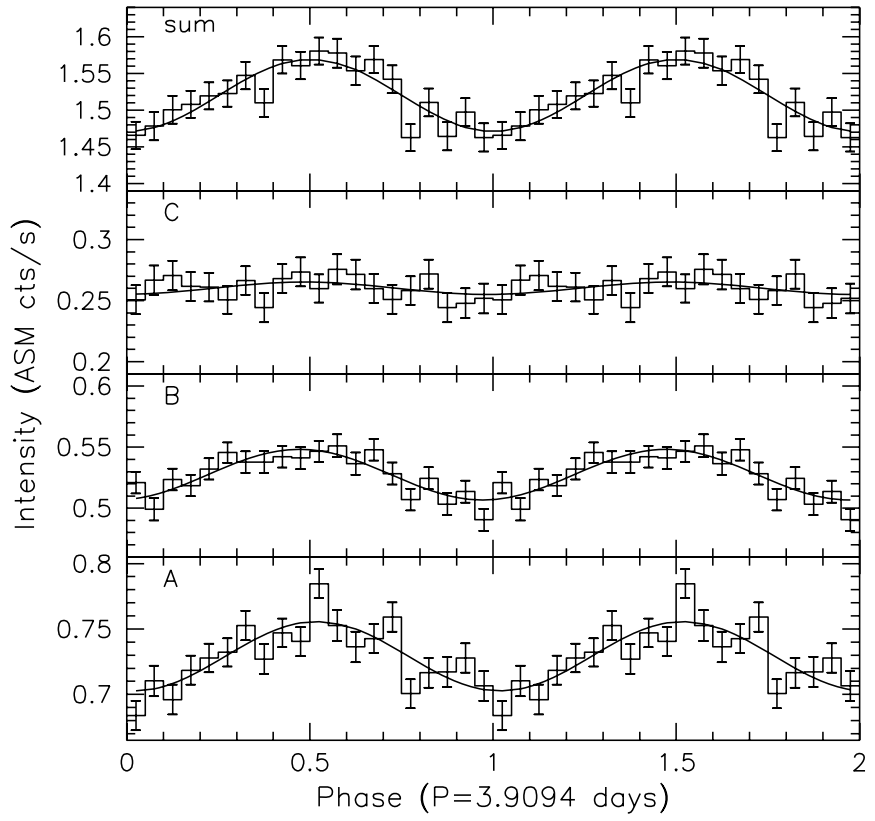

Figure 9. RXTE ASM light curves of LMC X-1 folded using a period of 3.9094 days and an epoch of phase zero of MJD 53390.75174. The four panels from bottom to top show the data from the $A(1.5-3 \mathrm{keV}), B$ (3$5 \mathrm{keV})$, and $C(5-12 \mathrm{keV})$ bands, and the sum of the three bands (nominally comprising photon energies $1.5-12 \mathrm{keV}$ ). The error bars indicate $\pm 1 \sigma$ statistical uncertainties. The smooth curves are the best-fit functions of the form $f(\phi)=$ $a_{0}+a_{1} \cos (2 \pi \phi)+a_{2} \sin (2 \pi \phi)$ (see Table 1 for the best-fitting parameters).

used in the smoothing was a Gaussian with a full width at halfmaximum of 30.0 days, so the smoothed light curve displayed only that variability with Fourier components at frequencies below $\approx 0.03 \mathrm{day}^{-1}$. The smoothed light curve was subtracted from the unsmoothed light curve, and the difference light curve was Fourier transformed. The results are illustrated in Figure 8. The center frequency of the peak and the formal uncertainty thereof are $0.25580 \pm 0.00005 \mathrm{day}^{-1}$. The corresponding period is $3.9094 \pm 0.0008$. This period differs from the optical period by only $1.3 \times 10^{-4}$ days.

In Figure 9 we show folded X-ray light curves for the 1.5$3,3-5$, and $5-12 \mathrm{keV}$ photon-energy bands as well as for the overall $1.5-12 \mathrm{keV}$ band. There is a clear orbital modulation with the minimum intensity occurring at phase 0 , which corresponds to the time of the inferior conjunction of the secondary star. The X-ray light curves can be fitted to a function of the form $f(\phi)=a_{0}+a_{1} \cos (2 \pi \phi)+a_{2} \sin (2 \pi \phi)$. Table 1 gives the bestfitting coefficients for the three individual bands and for the sum. In all cases, the $a_{2}$ terms are consistent with zero. We discuss this modulation in more detail in Section 5.3.

\subsection{Temperature and Rotational Velocity}

The effective temperature of the secondary star and its (projected) rotational velocity are needed as inputs and constraints on the dynamical model discussed below. Previously, Hutchings et al. (1983) derived a spectral type of O7 for the secondary star based on the line strengths of $\mathrm{He}$ I, $\mathrm{He}$ II, Si IV, and Mg II and a rotational velocity of $V_{\text {rot }} \sin i \approx 150 \mathrm{~km} \mathrm{~s}^{-1}$. Negueruela \& Coe (2002) derived a spectral type of O8III based on the ratio of the He II $\lambda 4541$ and He I $\lambda 4471$ lines and on the strength of the $\mathrm{Mg}$ II $\lambda 4481$ line. A spectral type of O8III corresponds to an effective temperature of around 33,000 K (Heap et al. 2006).

We used model spectra from the OSTAR2002 grid (Lanz \& Hubeny 2003) to derive improved values of the temperature 


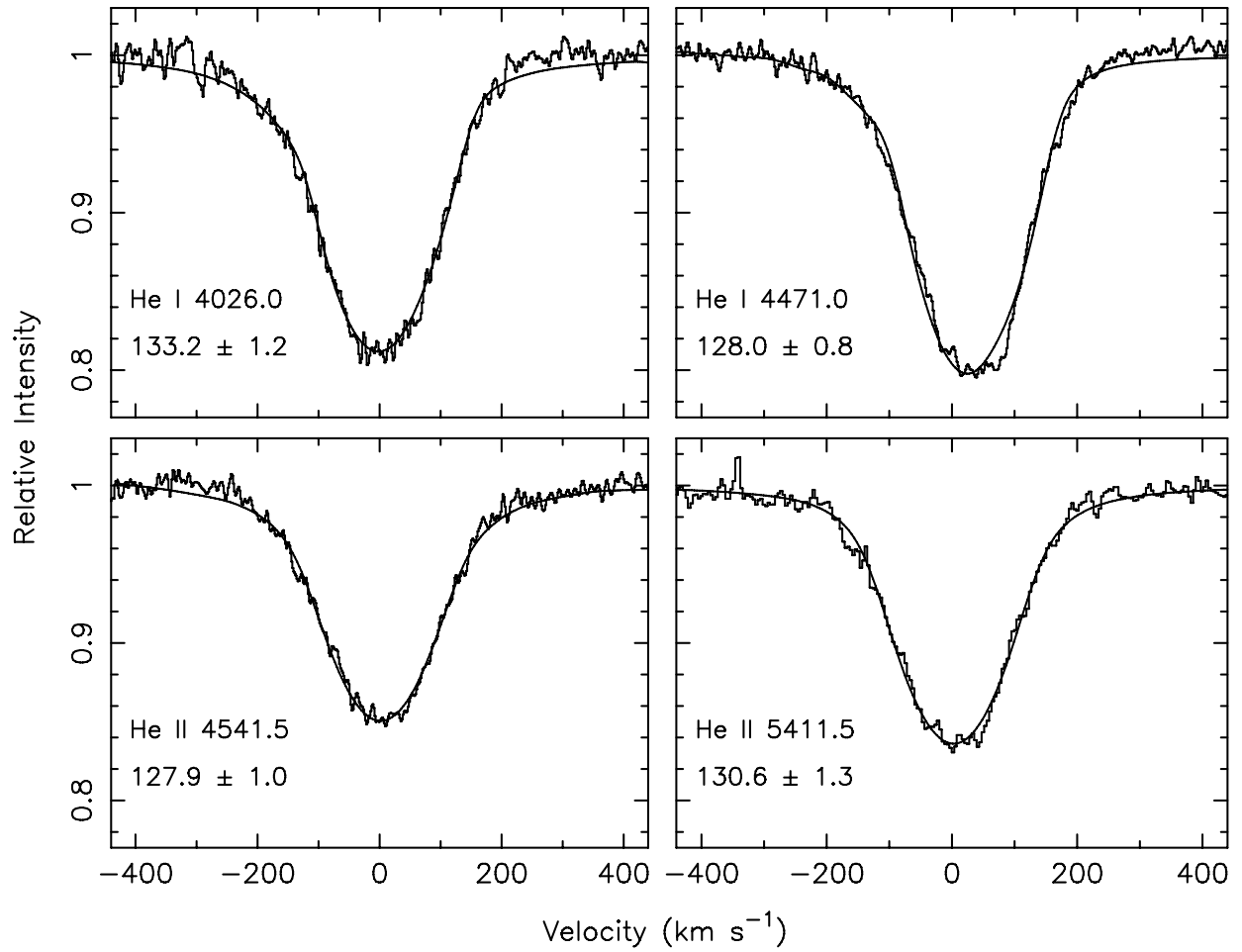

Figure 10. Average profiles of the four helium lines used to determine the rotational velocity ("histograms"), and the best-fitting model profiles for each (smooth curves). The line identification and the derived value of $V_{\text {rot }} \sin i$ in $\mathrm{km} \mathrm{s}^{-1}$ are given in each panel.

Table 1

Fitting Parameters for ASM Light Curves ${ }^{\mathrm{a}}$

\begin{tabular}{lccccc}
\hline \hline Channel & $a_{0}$ & $a_{1}$ & $a_{2}$ & $\chi_{v}^{2}$ & $A^{\mathrm{b}}$ \\
\hline$A$ & $0.7291 \pm 0.0025$ & $-0.0263 \pm 0.0036$ & $-0.0031 \pm 0.0035$ & 1.942 & $0.072 \pm 0.010$ \\
$B$ & $0.5275 \pm 0.0020$ & $-0.0204 \pm 0.0028$ & $0.0037 \pm 0.0028$ & 1.151 & $0.077 \pm 0.011$ \\
$C$ & $0.2602 \pm 0.0026$ & $-0.0050 \pm 0.0038$ & $0.0005 \pm 0.0037$ & 0.681 & $0.038 \pm 0.029$ \\
SUM & $1.5201 \pm 0.0042$ & $-0.0491 \pm 0.0060$ & $-0.0004 \pm 0.0059$ & 1.236 & $0.065 \pm 0.008$
\end{tabular}

Notes.

${ }^{\mathrm{a}} f(\phi)=a_{0}+a_{1} \cos (2 \pi \phi)+a_{2} \sin (2 \pi \phi)$

${ }^{\mathrm{b}}$ Fractional amplitude $A=\left(2\left|a_{1}\right| / a_{0}\right)$.

and rotational velocity of the secondary. The temperature and gravity sampling of the grid was extended to $50 \mathrm{~K}$ and 0.05 dex, respectively, by first resampling the high dispersion model spectra to a fine wavelength spacing common to all models and then by interpolation of the resampled models. Hubeny's program ROTINS3 was used to broaden model spectra with instrumental and rotational broadening profiles, and to resample the resulting models to match the wavelength sampling of the observed spectra.

The "surface" gravity of the secondary star is tightly constrained by the dynamical solution discussed below and is found to be $\log g=3.50$ with a formal $1 \sigma$ error less than 0.02 dex. For the purposes of finding the temperature we allow $\log g$ to fall within the generous range of $3.4 \leqslant \log g \leqslant 3$.6. With our large grid of model spectra over a large range of temperatures, gravities, and rotational velocities, we performed $\chi^{2}$ tests using nine helium lines between $4000 \AA$ and $5020 \AA$. The line ratios of $\mathrm{He}$ II $\lambda 4541 / \mathrm{He}$ I $\lambda 4471$ and $\mathrm{He}$ II $\lambda 4200 / \mathrm{He}$ I (+II) $\lambda 4026$, which are the main classification criteria for O-stars (e.g., Walborn \& Fitzpatrick 1990), are included in this range. We find a temperature of $T_{2}=33,225 \pm 75 \mathrm{~K}$ from the average MIKE spectrum and $T_{2}=33,200 \pm 150 \mathrm{~K}$ from the average MagE spectrum. Figure 3 shows the best-fitting model with the MIKE spectrum. Generally the lines in the model spectrum match up with the lines in the observed spectrum. There are a few exceptions such as the $\mathrm{O}$ II $+\mathrm{C}_{\text {III }}$ blend near $4035 \AA$, the Bowen blend near $4640 \AA$, the He II line near $4686 \AA$, and the $\mathrm{C}$ III line near $5690 \AA$. The wings and the cores of the Balmer lines are not well fitted. However, in the case of the MIKE spectra it is difficult to normalize the profiles of the Balmer lines owing to their large widths which can take up a substantial part of an individual order. The Balmer lines are matched better in the MagE spectrum where it is much easier to normalize the individual orders owing to the lower spectral resolution.

The errors on the temperature quoted above are the formal $1 \sigma$ errors. The true uncertainty on the effective temperature is no doubt larger owing to the fact that the model spectrum is not a perfect fit to the data. Also, there are almost certainly systematic errors in the model atmospheres as well. Accordingly, we will adopt a temperature of $T_{2}=33,200 \mathrm{~K}$ with an uncertainty of $500 \mathrm{~K}$ for the analysis presented below.

To refine our initial measurement of the rotational velocity of the star, we used the He I lines near $4026.0 \AA$ and 4471.0 $\AA$ and the He II lines near $4541.4 \AA$ and $5411.4 \AA$. We computed large grids of models with various temperatures, gravities, and rotational velocities with the appropriate resolving power 
Table 2

LMC X-1 Column Density Measurements

\begin{tabular}{lccccc}
\hline \hline Model Number & Model & Mission & $N_{\mathrm{H}}\left(10^{21} \mathrm{~cm}^{-2}\right)$ & $A_{V}(\mathrm{mag})$ & Reference \\
\hline 1 & DBB & Chandra HETG & $4.6 \pm 0.2$ & $2.57 \pm 0.12$ & Cui et al. 2002 \\
2 & COMPTT & Chandra HETG & $5.9 \pm 0.3$ & $3.30 \pm 0.18$ & Cui et al. 2002 \\
3 & DBB & BeppoSAX & $8.1 \pm 0.5$ & $4.53 \pm 0.29$ & Haardt et al. 2001 \\
4 & GRdisk & ASCA & $5.3 \pm 0.2$ & $2.96 \pm 0.12$ & Gierliński et al. 2001 \\
5 & DBB & ASCA & $6.3 \pm 0.1$ & $3.52 \pm 0.08$ & Nowak et al. 2001 \\
6 & DBB & BBXRT & $5.8 \pm 0.9$ & $3.24 \pm 0.51$ & Schlegel et al. 1994 \\
7 & X-ray halo & Chandra & $6.5 \pm 0.1$ & $3.65 \pm 0.10$ & Xiang et al. 2005 \\
\hline
\end{tabular}

for the MIKE spectrograph and performed $\chi^{2}$ tests to the composite line profiles (here we assume that the main broadening mechanism in the line profiles is rotation). Figure 10 shows the results. The model profiles fit the observed profiles fairly well. The rotational velocities found from the individual lines range from $127.9 \pm 1.0 \mathrm{~km} \mathrm{~s}^{-1}$ to $133.2 \pm 1.2 \mathrm{~km} \mathrm{~s}^{-1}$, where the uncertainties are the formal $1 \sigma$ errors. The average value is $129.9 \mathrm{~km} \mathrm{~s}^{-1}$, and the standard deviation of the four measurements is $2.18 \mathrm{~km} \mathrm{~s}^{-1}$. Hence for the analysis below, we adopt a value of $V_{\text {rot }} \sin i=129.9 \pm 2.2 \mathrm{~km} \mathrm{~s}^{-1}$.

\subsection{Radius of the Secondary Star and Reddening}

Unlike the case for most Galactic X-ray binaries, the distance to LMC X-1 is well determined. Given the distance, we can find the radius of the secondary star if we can obtain good measurements of the apparent magnitude of the star, its effective temperature, the extinction, and the bolometric correction. If the radius of the secondary star can be found independently, then the dynamical model of the system is further constrained.

The apparent magnitude of the system is easy to measure. The spectral type of the star yields its effective temperature, and the bolometric corrections can be estimated using detailed model atmosphere computations (e.g., Lanz \& Hubeny 2003; Martins \& Plez 2006). The remaining quantity, the extinction to the source, is unfortunately subject to the largest uncertainties and difficult to measure.

Using spectra from the International Ultraviolet Explorer (IUE), Bianchi \& Pakull (1985) determined a color excess of $E(B-V)=0.37$ (0.32 from extinction in the LMC and 0.05 Galactic foreground extinction). Bianchi \& Pakull (1985) noted that the color excess derived from the IUE data is lower than the value derived from the optical color of the star. Their value of $B-V=0.29$ and $(B-V)_{0}=-0.27$ (Martins \& Plez 2006) gives $E(B-V)=0.56$. Using our measured value of $B-V=0.17$ we find $E(B-V)=0.44$. Thus we have a range of color excess values of $0.37 \leqslant E(B-V) \leqslant 0.56$. Using $A_{V}=R_{V} E(B-V)$ with $R_{V}=3.1$ (the mean value for the LMC; Cardelli et al. 1989), the $V$-band extinction would be in the range of $1.15 \leqslant A_{V} \leqslant 1.74$.

These values of $A_{V} \leqslant 1.74 \mathrm{mag}$ are at odds with the extinction derived from two other techniques. In the first case, from our photometry we find a $V-K$ color of $V-K=$ $1.17 \pm 0.05$. O-stars, including dwarfs, giants, and supergiants of O-subtypes 6 through 9 , have $V-K$ colors in a relatively narrow range of $-0.87 \leqslant V-K \leqslant-0.83$ (Martins \& Plez 2006). Assuming $A_{K}=0.11 A_{V}$ (Cardelli et al. 1989), we need $A_{V}=2.28 \pm 0.06$ to match the observed $V-K$ color. In the second case, the $V$-band extinction can be inferred from the hydrogen column densities derived from X-ray spectra. Table 2 gives seven determinations of the column density $N_{\mathrm{H}}$ and the inferred $V$-band extinction, assuming $A_{V}=N_{\mathrm{H}} /(1.79 \pm$
$0.03) \times 10^{21}$, where $N_{\mathrm{H}}$ is given in units of $\mathrm{cm}^{-2}$ (Predehl \& Schmitt 1995). The values of the extinction cover a considerable range: $2.57 \pm 0.12 \leqslant A_{V} \leqslant 4.53 \pm 0.29$. We believe the first measurement given in the table $\left(A_{V}=2.57 \pm 0.12\right.$ from Cui et al. 2002) is the most reliable given the model used (the multitemperature disk blackbody model) and the data (high-resolution grating spectra from Chandra HETG). This measurement differs by the extinction derived from the $V-K$ color by only $2.5 \sigma$. Furthermore, we note that the X-ray column density may systematically exceed the value inferred from optical reddening (i.e., dust) considerations, if even a fraction of the dense stellar wind from the O-star is not completely ionized.

Adopting $A_{V}=2.28 \mathrm{mag}$, the range of color excess values quoted above $(0.37 \leqslant E(B-V) \leqslant 0.56)$ gives $4.07 \leqslant R_{V} \leqslant$ 6.16. Our measured value of color excess of $E(B-V)=0.44$ gives $R_{V}=5.18$. The nominal average value in the LMC is $R_{V}=3.1$, although certain lines of sight can have $R_{V}$ values that differ significantly from the average (Cardelli et al. 1989).

To compute the radius of the star, we assume a distance modulus to the LMC of $18.41 \pm 0.10$ (see Orosz et al. 2007). Although the uncertainty of the distance modulus to the center of the LMC itself is somewhat smaller than 0.1 mag, we adopt an uncertainty of $0.1 \mathrm{mag}$ to account for the unknown relative position of $\mathrm{LMC} \mathrm{X-1}$ with respect to the $\mathrm{LMC}$ center. We use bolometric corrections for the $V$ and $K$ bands derived from the OSTAR2002 models with the LMC mean metallicity (Lanz \& Hubeny 2003). ${ }^{11}$ We use apparent magnitudes of $V=14.6 \pm 0.05$ and $K=13.43 \pm 0.03$ (where we have increased the uncertainties to be conservative), and assume the star is the only source of optical light in the system (see the discussion in the Appendix regarding the likely faintness of the accretion disk). We use an effective temperature of $T_{2}=$ $33,200 \pm 500 \mathrm{~K}$, and set the gravity to $\log g=3.50 \pm 0.02$, as determined from the dynamical model described below. We computed the stellar radius using the $V$-band magnitudes and $K$-band magnitudes using a wide range of values of $A_{V}$, and the results are shown in Figure 11. The lines cross near $A_{V} \approx 2.2 \mathrm{mag}$ (where $R_{2} \approx 17 R_{\odot}$ ), which is not at all surprising since we derived $A_{V}=2.28 \pm 0.06 \mathrm{mag}$ from the $V-K$ color above. Note that the radius derived from the $K$ band is relatively insensitive to the assumed value of $A_{V}$, owing to the fact that $A_{K}=0.11 A_{V}$. The radii found using the $K$ band are in the range $15.5 \lesssim R_{2} \lesssim 18.3 R_{\odot}$ (this includes the $1 \sigma$ errors) for extinction values in the generous range of $1.4 \leqslant A_{V} \leqslant 2.8 \mathrm{mag}$. For a given value of $A_{V}$, the uncertainty in the radius derived from the $V$ band is on the order of $1.0 R_{\odot}$

\footnotetext{
11 The OSTAR2002 bolometric corrections are in good agreement with the bolometric corrections given in Martins \& Plez (2006). We prefer the former since they are tabulated in terms of the effective temperature and gravity, although the bolometric corrections are insensitive to the gravity.
} 


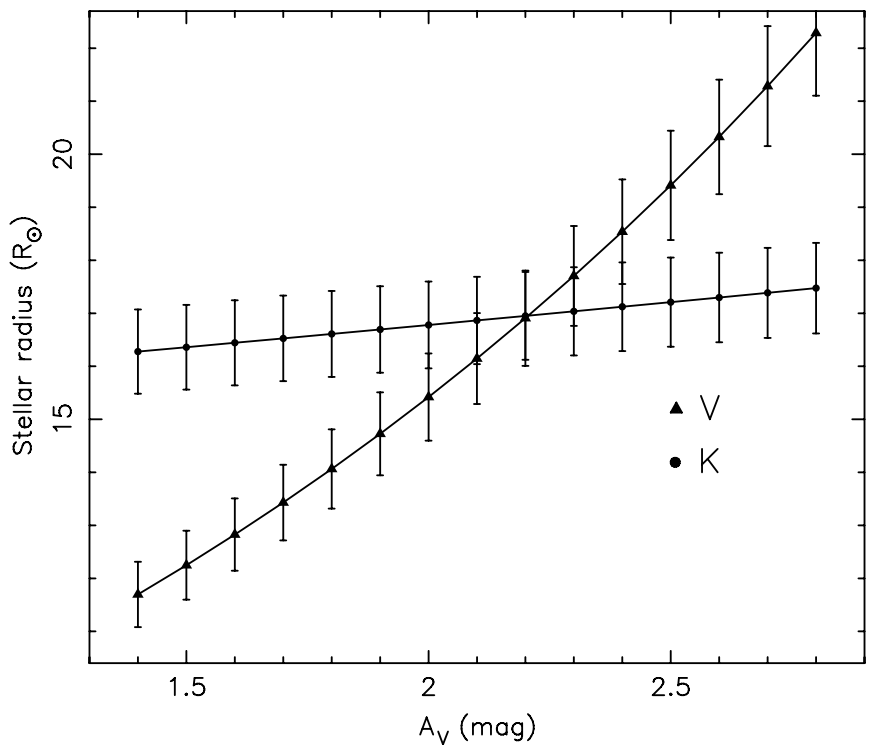

Figure 11. Radius of the companion as a function of the extinction $A_{V}$ for the $V$ band (triangles) and the $K$ band (circles). The radius is the same for both bands when the extinction is $\approx A_{V}=2.2 \mathrm{mag}$.

when the generous uncertainties in the apparent magnitudes and the large range in effective temperatures are used. For the $K$ band, the corresponding uncertainty is on the order of $0.8 R_{\odot}$. For the purposes of the dynamical model below we adopt $R_{2}=17.0 \pm 0.8 R_{\odot}$, which is the value we find using the $K$ band and $A_{V}=2.28 \pm 0.06$. The luminosity of the star is then $\log L / L_{\odot}=5.50 \pm 0.05$.

As noted above, the extinction derived from the IUE spectra is unusually low. In order to see if the IUE spectra could be explained by a different extinction law, we obtained the IUE spectra of LMC X-1 from the MAST archive. The spectra have been reprocessed using the latest calibrations, and we combined all of the observations to increase the signal-to-noise. The mean IUE spectrum was dereddened using $A_{V}=2.28$ and $R_{V}=$ 5.18. The results are shown in Figure 12. We also show a model spectrum from the OSTAR2002 grid with $T_{\text {eff }}=32,500 \mathrm{~K}$ and $\log g=3.5$, scaled using a distance modulus of 18.41 and a stellar radius of $17.0 R_{\odot}$. The agreement between the data and the model is good redward of the $2200 \AA$ bump. However, the $2200 \AA$ bump itself seems over-corrected, and the slope of the dereddened spectrum blueward of the bump is much too flat. However, the following caveats should be noted regarding the IUE data. First, the signal-to-noise of the spectra is not terribly high (on the order of 10 per pixel or less). Second, LMC X-1 was observed in the IUE large aperture $\left(10^{\prime \prime}\right)$, and the source was not centered in the aperture in order to exclude light from the much brighter star R148. Finally, Pakull \& Angebault (1986) showed that LMC X-1 is in a compact He III nebula, which is in turn embedded in a larger H II region known as N159 (Bianchi \& Pakull 1985). Considering the very large aperture of the IUE spectra, the UV spectrum of LMC X-1 might be contaminated by scattered light from nearby bright stars. It would be worthwhile to obtain additional UV spectra of LMC $\mathrm{X}-1$ to further investigate the UV extinction.

\subsection{System Parameters}

\subsubsection{Simultaneous Fits to Light and Velocity Curves}

We have several "observables" of the LMC X-1 binary system (e.g., the radial velocity curve of the secondary, its $B$ and $V$ light

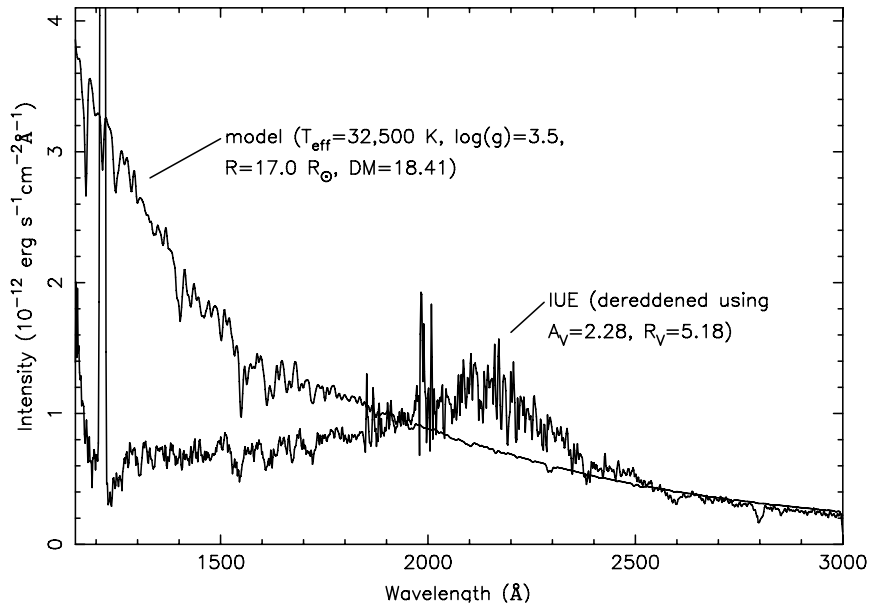

Figure 12. Average IUE spectrum of LMC X-1, dereddened using $A_{V}=2.27$ and $R_{V}=5.16$, is shown with a model spectrum from the OSTAR2002 grid having $T_{\text {eff }}=32,500 \mathrm{~K}$ and $\log g=3.5$, scaled using a distance modulus of 18.41 and a stellar radius of $17.08 R_{\odot}$.

curves, etc.), and we seek the physical model whose observed properties best match the observed data. While the "forward" problem of computing the observable properties of a binary system is relatively straightforward, the "inverse" problem of deriving a physical model from observed data is much more challenging. The ELC code (Orosz \& Hauschildt 2000) is a comprehensive code for computing the forward problem using a model based on Roche geometry. ELC can also solve the inverse problem using its associated optimizing codes based on various numerical techniques (e.g., a "grid search," an "amoeba," a Levenberg-Marquardt scheme, a genetic code, and a Monte Carlo Markov Chain scheme).

The ELC model as applied to LMC X-1 has several parameters, many of which can be set to reasonable values based on various observed properties of the system. The important parameters are related either to the geometrical properties of the system or to the radiative properties of the system.

The free parameters which control the basic system geometry include the orbital period $P$, the orbital separation $a$ (which then gives the total mass via Kepler's third law), the ratio of the masses $Q=M / M_{2}$, the inclination $i$, and the Roche lobe filling factor $f_{2}$ of the secondary star. We find that the search of parameter space is made more efficient if the $K$-velocity of the secondary star $K_{2}$ and the mass of the secondary star $M_{2}$ are used to set the system scale instead of $Q$ and $a$. When modeling actual data, one must also specify the phase zero point $T_{0}$, which is either the time of the inferior conjunction of the secondary star (in the case of a circular orbit) or the time of periastron passage (in the case of an eccentric orbit). We initially assume the secondary star is rotating synchronously with its orbit, and that the star's rotational axis is perpendicular to the orbital plane. We discuss in Section 4.4.2 how our results depend on the assumption of synchronous rotation.

The parameters which control the radiative properties include the average temperature of the secondary star $T_{2}$, its gravity darkening exponent $\beta$, and its bolometric albedo $A$. Following standard practice, we set $\beta=0.25$ and $A=1$, which are values appropriate for stars with a radiative envelope. ELC uses specific intensities derived from model atmosphere calculations, so no parameterized limb darkening law is needed. In modeling the LMC X-1 data, we use model atmospheres computed for the LMC metallicity from the OSTAR2002 grid (Lanz \& Hubeny 
Table 3

LMC X-1 Adopted Parameters

\begin{tabular}{lccc}
\hline \hline Parameter & $\begin{array}{c}\text { Value } \\
\text { (Circular Orbit) }\end{array}$ & $\begin{array}{c}\text { Value } \\
\text { (Eccentric Orbit) }\end{array}$ & $\begin{array}{c}\text { Adopted } \\
\text { Value }^{\mathrm{a}}\end{array}$ \\
\hline$P($ days $)$ & $3.90914 \pm 0.00005$ & $3.90917 \pm 0.00005$ & $3.90917 \pm 0.00005$ \\
$T_{0}(\mathrm{HJD} 2,453,300+)^{\mathrm{b}}$ & $91.3436 \pm 0.0078$ & $91.3419 \pm 0.0087$ & $91.3436 \pm 0.0080$ \\
$K_{2}\left(\mathrm{~km} \mathrm{~s}^{-1}\right)$ & $71.61 \pm 0.67$ & $70.74 \pm 0.75$ & $71.61 \pm 1.10$ \\
$i(\mathrm{deg})$ & $36.38 \pm 1.92$ & $37.00 \pm 1.78$ & $36.38 \pm 2.02$ \\
$f_{2}$ & $0.886 \pm 0.036$ & $0.894 \pm 0.040$ & $0.886 \pm 0.037$ \\
$M_{2}\left(M_{\odot}\right)$ & $31.79 \pm 3.48$ & $30.62 \pm 3.17$ & $31.79 \pm 3.67$ \\
$e$ & $\ldots$ & $0.0256 \pm 0.0066$ & $\ldots$ \\
$\omega($ deg $)$ & $\ldots$ & $260.5 \pm 16.8$ & $\ldots$ \\
$T_{0}(\mathrm{HJD} 2,453,300+)^{\mathrm{c}}$ & $\ldots$ & $91.3072 \pm 0.2073$ & $\ldots$ \\
$R_{2}\left(R_{\odot}\right)$ & $16.89 \pm 0.87$ & $16.41 \pm 0.71$ & $17.00 \pm 0.80^{\mathrm{d}}$ \\
$\log g(\mathrm{cgs})$ & $3.485 \pm 0.014$ & $3.497 \pm 0.011$ & $3.485 \pm 0.018$ \\
$a\left(R_{\odot}\right)$ & $36.49 \pm 1.42$ & $35.97 \pm 1.28$ & $36.49 \pm 1.51$ \\
$M\left(M_{\odot}\right)$ & $10.91 \pm 1.41$ & $10.30 \pm 1.18$ & $10.91 \pm 1.54$ \\
$\chi^{2}(B, V$ bands $)$ & $59.18,60.44$ & $59.00,59.70$ & $\ldots$ \\
$\chi^{2}($ radial velocities $)$ & 49.63 & 36.20 & $\ldots$ \\
$\chi^{2}$ (total) & 169.27 & 155.39 & $\ldots$ \\
\hline
\end{tabular}

Notes. The effective temperature of the secondary has been constrained to the range $32,200 \leqslant T_{\text {eff }} \leqslant 34,200 \mathrm{~K}$.

${ }^{a}$ The uncertainties include systematic errors.

${ }^{b}$ Time of inferior conjunction of secondary.

c Time of periastron passage of secondary.

${ }^{\mathrm{d}}$ Determined from the temperature, $K$ magnitude, distance, and extinction.

2003), supplemented by models from the BSTAR2006 grid at lower temperatures (Lanz \& Hubeny 2007).

ELC can include light from a flared accretion disk, and geometrical effects due to the disk (e.g., eclipses of the secondary star). If the disk contributes a significant amount of the light from the binary the observed amplitude of the ellipsoidal light curve will decrease. Thus it is critical to understand how luminous the accretion disk is relative to the star. We show in the Appendix that the accretion disk contributes at most $\approx 10 \%$ of the light in the optical and most likely contributes less than a few percent of the light. We therefore omit the disk in the models. We discuss in Section 4.4.2 how our results depend on the assumption of no disk light.

The effects of X-ray heating are computed using a scheme adapted from Wilson (1990). The source of the X-rays is assumed to be a thin disk with a radius that is very small compared to the orbital separation. The extent to which Xray heating changes the light (and velocity) curves depends primarily on how large the X-ray luminosity is compared to the bolometric luminosity of the secondary star. From our ongoing work on modeling 53 RXTE PCA X-ray spectra of LMC X-1, we find that the isotropic bolometric X-ray luminosity of LMC X-1 is quite steady over a time span of several years and is $\approx 2.3 \times 10^{38} \mathrm{erg} \mathrm{s}^{-1}$. For comparison, the bolometric luminosity of the star is $L \approx 1 \times 10^{39} \mathrm{erg} \mathrm{s}^{-1}$. Because the star intercepts only a modest fraction of the total X-ray flux, we thus expect that the effects of X-ray heating on the light and velocity curves will be minor. We show in Section 4.4.2 that our main results are insensitive to reasonable changes in $L_{x}$, and that systematic errors in the inclination and the derived masses due to improper treatment of X-ray heating are very small. In the following analysis we adopt $L_{x}=2.3 \times 10^{38} \mathrm{erg} \mathrm{s}^{-1}$.

To summarize, we have seven free parameters: $i, K_{2}, M_{2}, P$, $T_{0}, T_{2}$, and $f_{2}$. The data we model include $58 B$-band and 57 $V$-band measurements from SMARTS, and 37 radial velocity measurements. The $J$-band light curve from SMARTS proved to be too noisy to model. The uncertainties on the individual observations were scaled to give $\chi^{2} \approx N$ for each set separately. The mean of the error bars after the scaling are 0.012 mag for $B, 0.015 \mathrm{mag}$ for $V$, and $2.62 \mathrm{~km} \mathrm{~s}^{-1}$ for the radial velocities. Finally, we have three additional observational constraints that we include in the model "fitness" (see Orosz et al. 2002): the projected rotational velocity of the $\operatorname{star}\left(V_{\mathrm{rot}} \sin i=129.9 \pm 2.2\right.$ $\left.\mathrm{km} \mathrm{s}^{-1}\right)$, the radius of the secondary $\operatorname{star}\left(R_{2}=17.0 \pm 0.8 R_{\odot}\right)$, and the fact that the X-ray source is not eclipsed.

Parameter space was thoroughly explored by running ELC's genetic optimizer several times with the order of the parameters changed (this gives different initial populations while leaving the volume of the explored parameter space unchanged). The value of $T_{2}$ was confined to the $2 \sigma$ range $32,200 \leqslant T_{2} \leqslant 34,200 \mathrm{~K}$ as determined from the spectra, while the other parameters were given generous ranges. The final values of the parameters were refined using the grid search optimizer. The uncertainties on the fitted and derived parameters were found using the technique described in Orosz et al. (2002). Table 3 gives the fitted parameters, some derived parameters, and their uncertainties for our adopted circular orbit model and for an eccentric model discussed in more detail below. Figure 13 shows curves of $\chi^{2}$ versus various fitting and derived parameters of interest for the circular orbit model, from which one may read off the bestfitting value of a given parameter and the $1 \sigma, 2 \sigma$, and $3 \sigma$ confidence ranges. Each parameter is well constrained by the ellipsoidal model, with the exception of the mean temperature of the secondary star $T_{2}$. The fact that $T_{2}$ is not constrained by the ellipsoidal model is not surprising in this case (e.g., a single optical star in a noneclipsing system) and is not a cause for concern since the optical spectra constrain the temperature quite nicely. We discuss in Section 4.4.2 how our results depend on the adopted value of $T_{2}$ for a wide range of values. Figure 2 shows the folded light curves with the corresponding model curves, while the folded radial velocity curve and the corresponding model curves are shown in Figure 6. 

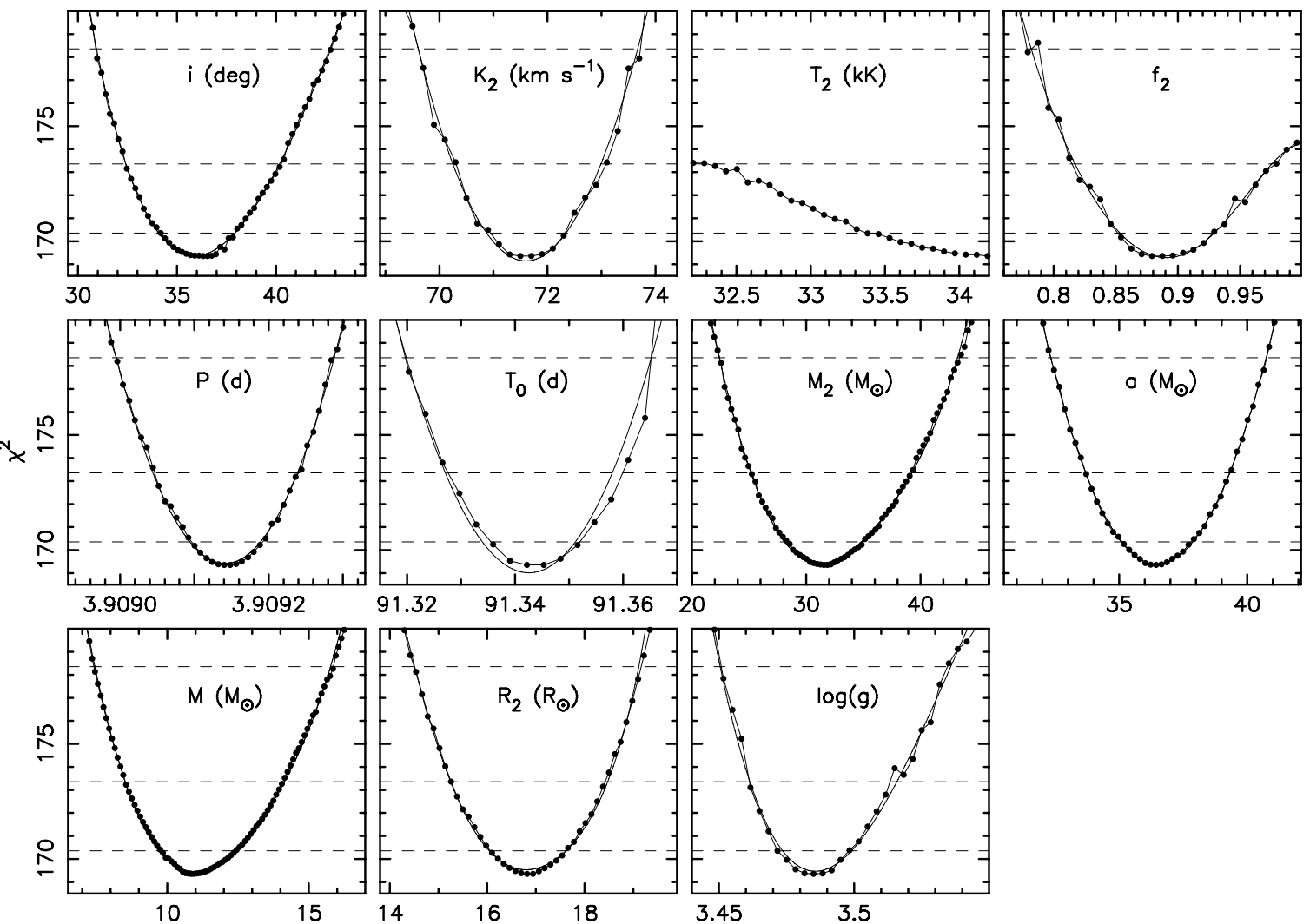

Figure 13. Curves of $\chi^{2}$ vs. various fitting and derived parameters of interest for the circular orbit model. The horizontal dashed lines denote the 1,2 , and $3 \sigma$ confidence limits.

\subsubsection{Possible Systematic Errors in the Light-Curve Models}

Our adopted parameters depend, among other things, an assumption that the orbit is circular and the star rotates synchronously with the orbit, and on two measured quantities that are not directly constrained (or only weakly constrained) by the light or velocity curves: the mean temperature of the secondary star $T_{2}$ and its radius $R_{2}$. The radius of the star, when used as an observed quantity, helps constrain the scale of the binary and hence the component masses. The temperature is needed to find the radius independently. We did numerical experiments to see how our results change when nonsynchronous rotation and eccentric orbits are allowed, when the input temperature of the star is changed, and when the radius (used as an extra constraint) is changed. We also explored how our results depend on our assumption that there is no disk light contamination in the optical and how our results change with different assumed values of the X-ray luminosity. But first, we briefly discuss the slight difference between the $K$-velocity derived from simple sinusoid fits to the radial velocities and the $K$-velocity found from the joint radial velocity and light-curve fits.

Systematic errors in $K_{2}$. The estimated $K$-velocity for LMC $\mathrm{X}-1$ depends slightly on which lines are used and how the spectra are normalized (Section 3.2 and Figure 4). A fourparameter sinusoid fit to the thirty seven radial velocities measured using all of the suitable lines yields an orbital period of $P=3.909139 \pm 0.000054$ days, and $K_{2}=69.79 \pm 0.65 \mathrm{~km}$ $\mathrm{s}^{-1}$, with $\chi^{2}=36.50$. This $K$-velocity is slightly smaller than the $K$-velocity found from the detailed modeling of the light and velocity curves $\left(K_{2}=71.61 \pm 0.67 \mathrm{~km} \mathrm{~s}^{-1}\right)$. (For comparison Hutchings et al. (1983) derived $K_{2}=65 \pm 7 \mathrm{~km} \mathrm{~s}^{-1}$ for a period of $P=3.909$ days.) There are two reasons for the difference.
The main reason is that the orbital phases of the radial velocities are tightly constrained when they are modeled together with the light curves. In this case, the time of the inferior conjunction of the secondary star found from a simple sinusoid fit to the velocities is slightly different from the same time found from the combined light and velocity curve modeling (see below). The second reason for the slight difference in the $K$-velocities is that ELC computes corrections to the radial velocities due to the distorted nature of the star, so the model curve is not precisely a sinusoid, even for a circular orbit.

Fitting the radial velocities along with the light curves provides a physical scale for the model. If one knew the $K$ velocity ahead of time, then one in principle could fit the light curves by themselves. However, since the $K$-velocity is not known exactly, it is better to use an additional $\chi^{2}$ constraint for $K_{2}$ rather than a fixed input value. We ran fits to the light curves by themselves using several values of $K_{2}$ as an extra constraint, assuming an uncertainty of $0.65 \mathrm{~km} \mathrm{~s}^{-1}$. The period was fixed at the value found from the four-parameter sinusoid fit, leaving six free parameters. The results are shown in Table 4. Not surprisingly, the masses scale as $K_{2}$. Since the computation of the light curves is not completely independent of the scale of the system (the model atmosphere specific intensities are tabulated in terms of the temperature and gravity in cgs units), there is a little bit of variation in the derived values of $i, T_{0}$, and $\chi^{2}$. The most important result contained in Table 4 is the fact that the derived values of the time of inferior conjunction of the secondary determined from the light curves is $\approx 2 \sigma$ different than that derived from the four-parameter sinusoid fit to the radial velocities. Since the photometrically determined time of conjunction should agree with the spectroscopically determined one, it is clearly better to fit the radial velocities together with 
Table 4

LMC X-1 Parameters as a Function of $K_{2}$

\begin{tabular}{lccccr}
\hline \hline $\begin{array}{l}\text { Assumed } K_{2} \\
\left(\mathrm{~km} \mathrm{~s}^{-1}\right)\end{array}$ & $\begin{array}{c}i \\
(\mathrm{deg})\end{array}$ & $\Delta \phi^{\mathrm{a}}$ & $\begin{array}{c}M_{2} \\
\left(M_{\odot}\right)\end{array}$ & $\begin{array}{c}M \\
\left(M_{\odot}\right)\end{array}$ & $\begin{array}{l}\chi_{\min }^{2} \\
68.50 \pm 0.65\end{array}$ \\
$36.20 \pm 2.02$ & $0.0111 \pm 0.0055$ & $31.46 \pm 3.51$ & $10.34 \pm 1.31$ & 115.68 \\
$69.50 \pm 0.65$ & $36.17 \pm 2.02$ & $0.0111 \pm 0.0057$ & $31.72 \pm 3.53$ & $10.58 \pm 1.33$ & 115.66 \\
$69.80 \pm 0.65$ & $36.17 \pm 2.03$ & $0.0111 \pm 0.0055$ & $31.79 \pm 3.48$ & $10.65 \pm 1.33$ & 115.65 \\
$70.50 \pm 0.65$ & $36.16 \pm 2.03$ & $0.0111 \pm 0.0055$ & $32.00 \pm 3.49$ & $10.82 \pm 1.36$ & 115.63 \\
$71.50 \pm 0.65$ & $36.23 \pm 2.01$ & $0.0112 \pm 0.0055$ & $32.10 \pm 3.51$ & $11.00 \pm 1.38$ & 115.60 \\
$72.50 \pm 0.65$ & $36.19 \pm 2.02$ & $0.0112 \pm 0.0055$ & $32.37 \pm 3.51$ & $11.26 \pm 1.41$ & 115.57 \\
\hline
\end{tabular}

Notes. The assumed distance is $18.41 \pm 0.10 \mathrm{mag}$, the assumed temperature is in the range $32,200 \leqslant T_{\text {eff }} \leqslant 34,200$ $\mathrm{K}$, and the assumed extinction is $A_{V}=2.28 \pm 0.06 \mathrm{mag}$.

a $\Delta \phi$ is the phase shift of the photometric $T_{0}$ relative to the spectroscopically determined value.

Table 5

LMC X-1 Parameters for Nonsynchronous Rotation

\begin{tabular}{|c|c|c|c|c|c|c|}
\hline$\overline{\Omega^{\mathrm{a}}}$ & $\begin{array}{c}i \\
(\mathrm{deg})\end{array}$ & $e$ & $\begin{array}{c}\omega \\
(\operatorname{deg})\end{array}$ & $\begin{array}{c}M_{2} \\
\left(M_{\odot}\right)\end{array}$ & $\begin{array}{c}M \\
\left(M_{\odot}\right)\end{array}$ & $\chi_{\min }^{2}$ \\
\hline 1.15 & $32.0 \pm 1.0$ & 0 (fixed) & $\ldots$ & $30.7 \pm 2.3$ & $12.5 \pm 1.0$ & 171.53 \\
\hline 1.10 & $33.0 \pm 1.4$ & 0 (fixed) & $\cdots$ & $31.4 \pm 2.4$ & $12.1 \pm 1.2$ & 170.45 \\
\hline 1.05 & $34.9 \pm 1.5$ & 0 (fixed) & $\cdots$ & $31.0 \pm 1.5$ & $11.3 \pm 1.4$ & 169.91 \\
\hline 1.00 & $36.4 \pm 1.9$ & 0 (fixed) & $\cdots$ & $31.2 \pm 3.5$ & $10.9 \pm 1.4$ & 169.38 \\
\hline 0.95 & $38.5 \pm 2.2$ & 0 (fixed) & $\cdots$ & $32.2 \pm 3.1$ & $10.3 \pm 1.0$ & 169.11 \\
\hline 0.90 & $42.8 \pm 2.5$ & 0 (fixed) & $\cdots$ & $31.6 \pm 2.9$ & $9.3 \pm 1.1$ & 168.73 \\
\hline 0.85 & $44.5 \pm 2.6$ & 0 (fixed) & $\cdots$ & $32.3 \pm 2.9$ & $8.4 \pm 0.9$ & 168.51 \\
\hline 0.80 & $48.1 \pm 2.6$ & 0 (fixed) & $\cdots$ & $33.0 \pm 2.2$ & $8.4 \pm 1.1$ & 168.18 \\
\hline 0.75 & $52.1 \pm 2.5$ & 0 (fixed) & $\cdots$ & $33.8 \pm 3.1$ & $7.9 \pm 0.9$ & 167.84 \\
\hline 0.70 & $56.3 \pm 2.7$ & 0 (fixed) & $\cdots$ & $35.6 \pm 2.9$ & $7.7 \pm 0.7$ & 168.17 \\
\hline 0.65 & $60.9 \pm 1.8$ & 0 (fixed) & $\cdots$ & $37.4 \pm 2.0$ & $7.5 \pm 0.4$ & 168.06 \\
\hline 1.15 & $33.8 \pm 1.5$ & $0.0215 \pm 0.0038$ & $267.0 \pm 17.2$ & $28.2 \pm 2.9$ & $11.0 \pm 1.2$ & 162.45 \\
\hline 1.10 & $34.6 \pm 1.7$ & $0.0210 \pm 0.0063$ & $265.5 \pm 16.4$ & $29.4 \pm 3.2$ & $11.0 \pm 1.3$ & 160.46 \\
\hline 1.05 & $36.0 \pm 1.8$ & $0.0245 \pm 0.0036$ & $261.3 \pm 16.8$ & $29.6 \pm 3.3$ & $10.7 \pm 1.4$ & 157.03 \\
\hline 1.00 & $37.4 \pm 2.1$ & $0.0269 \pm 0.0038$ & $266.3 \pm 16.9$ & $30.3 \pm 3.4$ & $10.3 \pm 1.4$ & 154.93 \\
\hline 0.95 & $39.4 \pm 2.0$ & $0.0287 \pm 0.0048$ & $266.3 \pm 17.2$ & $31.4 \pm 3.2$ & $9.8 \pm 1.1$ & 153.19 \\
\hline 0.90 & $42.1 \pm 1.7$ & $0.0293 \pm 0.0042$ & $264.5 \pm 16.6$ & $32.2 \pm 2.4$ & $9.2 \pm 0.9$ & 152.35 \\
\hline 0.85 & $44.2 \pm 2.2$ & $0.0311 \pm 0.0046$ & $265.2 \pm 17.0$ & $33.5 \pm 2.8$ & $9.0 \pm 1.2$ & 150.76 \\
\hline 0.80 & $47.6 \pm 2.6$ & $0.0336 \pm 0.0045$ & $266.2 \pm 17.5$ & $34.5 \pm 3.6$ & $8.6 \pm 1.1$ & 149.94 \\
\hline 0.75 & $50.3 \pm 3.5$ & $0.0360 \pm 0.0038$ & $268.3 \pm 17.1$ & $36.4 \pm 3.5$ & $8.4 \pm 1.0$ & 149.48 \\
\hline 0.70 & $56.8 \pm 4.0$ & $0.0372 \pm 0.0035$ & $268.2 \pm 16.5$ & $36.1 \pm 3.9$ & $7.5 \pm 0.9$ & 148.32 \\
\hline 0.65 & $61.7 \pm 2.6$ & $0.0372 \pm 0.0037$ & $259.9 \pm 17.0$ & $38.0 \pm 2.4$ & $7.4 \pm 0.4$ & 148.46 \\
\hline
\end{tabular}

Notes. The temperature is in the range $32,200 \leqslant T_{\text {eff }} \leqslant 34,200 \mathrm{~K}$, the assumed distance is $18.41 \pm 0.10 \mathrm{mag}$, and the assumed extinction is $A_{V}=2.28 \pm 0.06 \mathrm{mag}$.

a $\Omega$ is the ratio of the rotation frequency of the star to the orbital frequency.

the light curves. The $K$-velocity derived from the simultaneous fit will be closer to the actual value, but at the expense of a larger $\chi^{2}$ value contribution from the radial velocities.

Possible nonzero eccentricity and nonsynchronous rotation. Although the timescales for a close binary to evolve to a circular orbit and synchronous rotation are relatively short, we should nevertheless entertain the possibility that the orbit of LMC $\mathrm{X}-1$ could be slightly eccentric and/or the rotation could be nonsynchronous because the age of the massive secondary star in LMC X-1 might be less than the timescales for synchronization or circularization. Also, some examples of high mass X-ray binaries with eccentric orbits are known and include Vela X-1 (Rappaport et al. 1976; Bildsten et al. 1997) and M33 X-7 (Orosz et al. 2007). Having an eccentric orbit adds the eccentricity $e$ and the argument of periastron $\omega$ as free parameters (see Avni 1976 and Wilson 1979 for a discussion of generalized Roche potentials for eccentric orbits). As before, parameter space was thoroughly explored by using the genetic optimizer, and the results are shown in Table 3.
Avni (1976) also discussed the generalization of the Roche potential for nonsynchronous rotation. The figure of the star depends somewhat on how fast it is rotating, and a convenient parameterization of the rotation rate is $\Omega$, which is the ratio of the rotation frequency of the star to the orbital frequency. For a circular orbit and synchronous rotation, we have $\Omega=1$. For an eccentric orbit, the idea of synchronous rotation is somewhat harder to define because the angular orbital speed of the star changes over its orbit. One can compute $\Omega$ so that the rotation frequency of the star matches its orbital frequency at periastron (Hut 1981). We computed models for a wide range of $\Omega$ for both circular and eccentric orbits, and the results are given in Table 5.

The eccentricity is formally nonzero at the $\approx 3 \sigma$ level since the total $\chi^{2}$ of the fit decreased by about 14 with the addition of two more free parameters. However, there are several reasons for believing that the nonzero eccentricity is spurious. (1) The sampling of the radial velocities is such that a best-fit sinusoid is shifted in phase relative to the light curves. In an eccentric 
Table 6

LMC X-1 Parameters as a Function of Temperature

\begin{tabular}{lcccccc}
\hline \hline $\begin{array}{l}\text { Temperature } \\
(\mathrm{K})\end{array}$ & $\begin{array}{c}\text { Derived Radius } \\
\left(R_{\odot}\right)\end{array}$ & $\begin{array}{c}\log L \\
\left(L_{\odot}\right)\end{array}$ & $\begin{array}{c}i \\
(\mathrm{deg})\end{array}$ & $\begin{array}{c}M_{2} \\
\left(M_{\odot}\right)\end{array}$ & $\begin{array}{c}M \\
\left(M_{\odot}\right)\end{array}$ & $\chi_{\min }^{2}$ \\
\hline 30,000 & $17.03 \pm 0.83$ & $5.33 \pm 0.04$ & $36.29 \pm 2.10$ & $32.34 \pm 3.48$ & $11.09 \pm 1.34$ & 174.14 \\
31,000 & $16.89 \pm 0.82$ & $5.38 \pm 0.04$ & $36.47 \pm 2.11$ & $31.93 \pm 1.6$ & $10.95 \pm 1.34$ & 174.66 \\
32,000 & $17.07 \pm 0.83$ & $5.44 \pm 0.04$ & $36.12 \pm 2.03$ & $32.73 \pm 3.56$ & $11.21 \pm 1.35$ & 173.94 \\
33,000 & $17.07 \pm 0.82$ & $5.49 \pm 0.04$ & $36.30 \pm 2.02$ & $32.45 \pm 3.73$ & $11.07 \pm 1.27$ & 171.30 \\
34,000 & $16.72 \pm 0.81$ & $5.53 \pm 0.04$ & $37.11 \pm 2.17$ & $30.67 \pm 3.61$ & $10.44 \pm 1.32$ & 169.24 \\
35,000 & $16.23 \pm 0.78$ & $5.55 \pm 0.04$ & $38.42 \pm 2.33$ & $28.66 \pm 3.21$ & $9.66 \pm 1.25$ & 169.55 \\
36,000 & $15.79 \pm 0.76$ & $5.58 \pm 0.04$ & $39.92 \pm 2.13$ & $27.12 \pm 3.00$ & $8.96 \pm 1.13$ & 170.07 \\
37,000 & $15.42 \pm 0.74$ & $5.61 \pm 0.04$ & $40.16 \pm 1.71$ & $26.81 \pm 1.91$ & $8.79 \pm 0.75$ & 165.82 \\
\hline
\end{tabular}

Note. The assumed distance is $18.41 \pm 0.10 \mathrm{mag}$ and the assumed extinction is $A_{V}=2.28 \pm 0.06 \mathrm{mag}$.

orbit, the phases of the minimum or the maximum velocity are shifted relative to a sine curve (see Figure 6), so that in the combined light + velocity curve modeling the total $\chi^{2}$ can be lower. Indeed, the $\chi^{2}$ value of the fit to the radial velocities for the eccentric model (Table 3 ) in the combined light + velocity curve analysis is comparable to the $\chi^{2}$ of the four-parameter best-fit sinusoid fit discussed above. In other words, an eccentric orbit fitted to the radial velocities alone is not significantly better than a simple sine curve fit. (2) The argument of periastron $\omega$ is consistent with $270^{\circ}$. This gives one pause because tidal distortions of the secondary can give rise to distorted line profiles, which in turn give rise to systematic errors in the measured radial velocities. These velocity errors can result in fits with spurious eccentricities and with the argument of periastron at a quadrature phase (e.g., Wilson \& Sofia 1976; Eaton 2008). ELC does compute corrections to the radial velocities in the manner of Wilson \& Sofia (1976), but these corrections may not be completely accurate. (3) When nonsynchronous models are considered (Table 5) we find that the eccentricity is correlated with the parameter $\Omega$. As the value of $\Omega$ gets smaller (i.e., as the star rotates slower and slower), the eccentricity and the inclination get larger. In fact, values of $\Omega$ smaller than about 0.65 are not possible since the best-fitting inclination would produce an X-ray eclipse, which is not observed at a high level of confidence. Hence, we would have for the best-fitting model in Table 5 a star rotating at $\approx 65 \%$ of the synchronous value in a moderately eccentric orbit where the X-ray source passes just below the limb of the star as seen in the plane of the sky. If we ignored the fact that there is no X-ray eclipse, our best-fitting model would have $i=90^{\circ}$. (4) The differences between the model light curves for the eccentric orbit model and the circular orbit model are quite subtle. (5) Detailed model computations specific to LMC X-1 give timescales for synchronization and circularization that are both a few orders of magnitude shorter than the lifetime of the secondary star for most values of the initial rotation rate (Valsecchi, Willems, \& Kalogera 2008, private communication).

Given these arguments which cast doubt on the reality of a nonzero eccentricity, we adopt the parameters derived from the circular orbit model. To be conservative with the uncertainties, the small differences between the parameters derived from the circular orbit model and the eccentric orbit model are taken to be a measure of the systematic errors. These systematic errors are added in quadrature to the uncertainties found for the circular orbit model to produce the final adopted uncertainties (the fourth column in Table 3 ).

Regarding the models for nonsynchronous rotation, some trends are evident in Table 5. First, as $\Omega$ goes from high values to lower values, the mass of the secondary star and the orbital increase, and the mass of the black hole decreases (i.e., the mass ratio $Q$ decreases). The reason for the mass ratio change is easy to understand. One can show that the rotational velocity of a star that fills its Roche lobe and is in synchronous rotation depends on the mass ratio (Wade \& Horne 1988). The same holds true for a star that underfills its Roche lobe, although the function relating the velocity to the mass ratio becomes more complicated. When the rotation is nonsynchronous, the relation between the velocity and the mass ratio changes further. The reason for the change in the inclination is also easy to understand. As the value of $\Omega$ decreases (i.e., as the star rotates more slowly), the star becomes less distorted. As a result, higher inclinations are needed to get roughly the same amplitudes for the light curves.

Finally, as the value of $\Omega$ goes down, the value of $\chi^{2}$ for the fit goes down as well. However, the change in $\chi^{2}$ over the entire range of $\Omega$ for the circular orbit is modest $\left(\Delta \chi^{2} \approx 3.5\right)$, whereas for the eccentric orbit the change is much larger $\left(\Delta \chi^{2} \approx 14\right)$. We have already discussed why we believe that the nonzero eccentricity may be spurious. For the circular orbit model, we have no strong observational constraint on the value of $\Omega$. Based on other considerations, we give two reasons why we believe $\Omega=1$. First, our formal best solution corresponds to a near-grazing X-ray eclipse which, although possible, seems unlikely. Second, as discussed above, the timescales for both synchronization and circularization are much shorter than the age of the secondary star for most initial conditions. We conclude that $\Omega$ is most likely to have a value very close to unity.

Fixed temperature. In our next experiment, we imagine that the temperature of the secondary is known exactly. In this case, the bolometric correction is known exactly (to the extent that one believes the model atmosphere computations). Once the bolometric correction is known, the radius of the star can be found using the apparent $K$ magnitude, the distance, and the extinction $A_{V}$. We ran eight simulations where the temperature was fixed at values between $30,000 \mathrm{~K}$ and $37,000 \mathrm{~K}$ in steps of $1000 \mathrm{~K}$. We assumed a distance modulus of $18.41 \pm$ $0.10 \mathrm{mag}$ and an extinction of $A_{V}=2.28 \pm 0.06 \mathrm{mag}$. Table 6 gives the derived radii and luminosities for these eight cases. For each case, we fit the data using the genetic code, assuming the orbit is circular. Table 6 also gives the resulting values of the inclination $i$, secondary star mass $M_{2}$, the black hole mass $M$, and the $\chi^{2}$ of the fit. A few trends are obvious from Table 6 . First, as the temperature increases, the derived radius is roughly constant until $T=34,000 \mathrm{~K}$, after which it decreases. This is due in part to the way the bolometric corrections change 
Table 7

LMC X-1 Parameters as a Function of Radius

\begin{tabular}{lccccc}
\hline \hline $\begin{array}{l}\text { Radius } \\
\left(R_{\odot}\right)\end{array}$ & $\begin{array}{c}\text { Derived Luminosity } \\
\left(\log L / L_{\odot}\right)\end{array}$ & $\begin{array}{c}i \\
(\mathrm{deg})\end{array}$ & $\begin{array}{c}M_{2} \\
\left(M_{\odot}\right)\end{array}$ & $\begin{array}{c}M \\
\left(M_{\odot}\right)\end{array}$ & $\chi_{\min }^{2}$ \\
\hline 15.0 & $5.39 \pm 0.03$ & $41.24 \pm 0.87$ & $24.56 \pm 0.95$ & $8.15 \pm 0.25$ & 168.52 \\
15.5 & $5.42 \pm 0.03$ & $39.64 \pm 0.80$ & $26.34 \pm 0.90$ & $8.86 \pm 0.28$ & 168.71 \\
16.0 & $5.45 \pm 0.03$ & $38.17 \pm 0.81$ & $28.17 \pm 0.97$ & $9.62 \pm 0.29$ & 168.99 \\
16.5 & $5.47 \pm 0.03$ & $36.82 \pm 0.71$ & $30.16 \pm 0.89$ & $10.43 \pm 0.28$ & 169.19 \\
17.0 & $5.50 \pm 0.03$ & $35.62 \pm 0.71$ & $32.28 \pm 0.97$ & $11.29 \pm 0.31$ & 169.44 \\
17.5 & $5.53 \pm 0.03$ & $34.42 \pm 0.70$ & $34.50 \pm 1.24$ & $12.22 \pm 0.30$ & 169.77 \\
18.0 & $5.55 \pm 0.03$ & $33.37 \pm 0.65$ & $36.86 \pm 0.87$ & $13.20 \pm 0.37$ & 170.42 \\
18.5 & $5.57 \pm 0.03$ & $32.39 \pm 0.56$ & $39.29 \pm 0.59$ & $14.22 \pm 0.34$ & 170.36 \\
19.0 & $5.56 \pm 0.03$ & $32.48 \pm 0.59$ & $42.60 \pm 0.56$ & $15.44 \pm 0.37$ & 171.27 \\
\hline
\end{tabular}

Note. The assumed temperature is in the range $32,200 \leqslant T_{\text {eff }} \leqslant 34,200 \mathrm{~K}$, the assumed distance is $18.41 \pm$ $0.10 \mathrm{mag}$, and the assumed extinction is $A_{V}=2.28 \pm 0.06 \mathrm{mag}$.

with temperature. The luminosity also increases with increasing temperature. Second, as the temperature increases, the $\chi^{2}$ of the fit generally goes down. On the other hand, the derived component masses do not change that much over the range of temperatures. The extreme values of the black hole mass are $8.79 \pm 0.75 M_{\odot}$ and $11.21 \pm 1.35 M_{\odot}$.

Fixed radius. Next, we can imagine that the radius of the secondary star is known exactly. In this case, we do not need to know either the distance to the source or the extinction. Using a temperature of $T_{2}=33,200 \pm 500 \mathrm{~K}$, we can compute the luminosity of the star and its uncertainty. We ran eight simulations. In each we fit the data using the genetic code (again using a circular orbit) with the secondary star radius fixed at one of the eight equally spaced values between $15.0 R_{\odot}$ and $19.0 R_{\odot}$. The results of this exercise (see Table 7 ) can be used to judge what would happen to our results if our adopted distance to the LMC is in error, or if our adopted value of the extinction $A_{V}$ were in error. For example, if the LMC were actually closer than what we assume, the radius that we would derive would be smaller (with all other things being equal). As before, a few trends are evident when inspecting Table 7. First, it is obvious that the luminosity of the star will go up as its radius goes up. As the radius increases, the scale of the binary increases leading to larger masses. As the star's radius goes up, it fills more of its Roche lobe, resulting in lower inclinations. In fact, good solutions with radii larger than $\approx 19.0 M_{\odot}$ are not possible since the star would then overfill its Roche lobe. Unlike the case when the temperature was fixed at various values, the $\chi^{2}$ of the fit changes very little when the radius is fixed at different values, except when $R_{2} \gtrsim 18 R_{\odot}$.

Variations in $X$-ray heating. As noted above, ELC assumes the source of X-rays is a thin disk with a small diameter compared to the orbital separation. Our value of $L_{x}(\approx 2.3 \times$ $10^{38} \mathrm{erg} \mathrm{s}^{-1}$, which includes the geometrical factor to account for the inclination angle of the disk as seen from Earth) may need two correction factors when used as an input to the simple way in which ELC computes the effects of X-ray heating. First, if there is extinction local to the source (for example, due to the outer edge of the accretion disk), some of the X-rays that would have reached the secondary star could be absorbed. If a flared accretion disk is present in the model, ELC can test which grid elements on the star are shielded from the X-ray source by the disk rim. However, it is hard to know what parameters to choose for the disk without more observational constraints. In any case, the first correction factor would be less than unity. The second correction factor comes from the fact that the X-ray source may not be a perfectly flat and thin disk that is in the orbital plane
Table 8

LMC X-1 Parameters as a Function of X-ray Heating

\begin{tabular}{lcccc}
\hline \hline $\begin{array}{l}\log L_{x}\left(\mathrm{erg} \mathrm{s}^{-1}\right) \\
\text { (Isotropic Equivalent) }\end{array}$ & $\begin{array}{c}i \\
(\mathrm{deg})\end{array}$ & $\begin{array}{c}M_{2} \\
\left(M_{\odot}\right)\end{array}$ & $\begin{array}{c}M \\
\left(M_{\odot}\right)\end{array}$ & $\chi_{\min }^{2}$ \\
\hline No heating & $36.02 \pm 1.78$ & $31.60 \pm 3.11$ & $10.98 \pm 1.28$ & 169.57 \\
38.00 & $36.02 \pm 1.82$ & $31.61 \pm 3.03$ & $10.99 \pm 1.40$ & 169.57 \\
38.25 & $36.01 \pm 1.28$ & $31.54 \pm 2.27$ & $10.99 \pm 0.99$ & 169.39 \\
38.50 & $36.06 \pm 1.83$ & $31.41 \pm 3.28$ & $10.95 \pm 1.40$ & 169.35 \\
38.75 & $36.02 \pm 1.39$ & $31.40 \pm 2.50$ & $10.97 \pm 0.77$ & 169.48 \\
39.00 & $35.91 \pm 1.77$ & $31.49 \pm 3.17$ & $11.05 \pm 1.16$ & 170.30
\end{tabular}

Note. The assumed temperature is in the range $32,200 \leqslant T_{\text {eff }} \leqslant 34,200 \mathrm{~K}$, the assumed distance is $18.41 \pm 0.10 \mathrm{mag}$, and the assumed extinction is $A_{V}=2.28 \pm 0.06 \mathrm{mag}$.

(i.e., the disk may be warped or there may be some kind of spherical corona that emits some of the X-rays). When there is a perfectly thin and flat disk, an observer on the star would see the X-ray emitting thin disk at an angle, and the intensity of X-rays observed at that point would be lower than it would be for the simple point source case owing to foreshortening of the disk (the foreshortening of the surface element itself is accounted for in the code). A warp in the disk or the presence of a quasi-spherical corona could increase the effective emitting area, leading to more X-rays hitting the secondary star. The second correction factor would be greater than unity.

To see how much the derived parameters depend on our adopted value of $L_{x}$, we ran nine numerical experiments with $\log L_{x}$ fixed at values between 38.0 and 39.0 in steps of 0.25 dex. We also set $L_{x}=0$ (e.g., the case for no X-ray heating). We assume a circular orbit, and that the free parameters and the ranges thereof were the same as in our main work. Table 8 gives the results. As the value of $L_{x}$ goes up, the $\chi^{2}$ of the fit remains virtually the same. In addition, there is little variation in the inclination and in the derived masses, and we conclude that our overall results are rather insensitive to our treatment of the X-ray heating.

Variations in the disk contribution. We show in the Appendix that the accretion disk contributes a few percent or less of the flux in the optical bands, and at most $\approx 10 \%$ when extreme ranges of parameters are considered. In the analysis above we assumed there was zero disk contribution. We ran models which included an accretion disk to see how our results depend on this assumption of zero disk contribution in the optical. In the ELC code one must specify the inner and outer disk radii, the disk opening angle, the temperature at the inner edge, and 
Table 9

LMC X-1 Parameters as a Function of the Disk Fraction

\begin{tabular}{lcccrc}
\hline \hline $\begin{array}{l}\text { Disk Fraction } \\
(V \text {-band })\end{array}$ & $\begin{array}{c}\text { Derived Radius } \\
\left(R_{\odot}\right)\end{array}$ & $\begin{array}{c}i \\
(\mathrm{deg})\end{array}$ & $\begin{array}{c}M_{2} \\
\left(M_{\odot}\right)\end{array}$ & $\begin{array}{c}M \\
\left(M_{\odot}\right)\end{array}$ & $\chi_{\min }^{2}$ \\
\hline 0.05 & $16.58 \pm 0.80$ & $37.31 \pm 1.84$ & $28.88 \pm 2.56$ & $10.03 \pm 1.10$ & 169.78 \\
0.10 & $16.15 \pm 0.80$ & $37.99 \pm 2.28$ & $27.25 \pm 2.65$ & $9.52 \pm 1.12$ & 170.16 \\
0.15 & $15.69 \pm 0.80$ & $38.44 \pm 1.43$ & $25.96 \pm 1.89$ & $9.15 \pm 0.73$ & 171.04 \\
0.20 & $15.22 \pm 0.80$ & $39.10 \pm 1.36$ & $24.43 \pm 1.70$ & $8.69 \pm 0.60$ & 175.73 \\
\hline
\end{tabular}

Notes. The assumed temperature is in the range $32,200 \leqslant T_{\text {eff }} \leqslant 34,200 \mathrm{~K}$, the assumed distance is $18.41 \pm$ $0.10 \mathrm{mag}$, and the assumed extinction is $A_{V}=2.28 \pm 0.06 \mathrm{mag}$.

the exponent on the power-law temperature profile. Since LMC $\mathrm{X}-1$ is not eclipsing, there is little to constrain the parameters of the accretion disk. Consequently we fixed the inner radius, the opening angle, and the exponent on the power-law temperature profile. The genetic code was run four times, and models where the disk fraction was within small threshold of a specified value were selected by means of an additional term in the total $\chi^{2}$. The various runs had the disk fraction in $V$ set at 0.05 , $0.10,0.15$, and 0.20 . If there is optical light from the accretion disk, then the O-star by itself would be less luminous since the apparent magnitude of the disk + star combination is fixed. As a result its computed radius would be smaller for a fixed temperature. Therefore in each of the four cases the assumed radius of the O-star was adjusted downward accordingly. The results are shown in Table 9. In general, as the disk fraction increases, the inclination increases slightly and the masses go down slightly. For a disk fraction of $10 \%$, the mass of the black hole decreases by $\approx 1 \sigma$. We therefore conclude that our results are relatively insensitive to our assumption of no disk contribution in the optical.

\section{DISCUSSION}

\subsection{The Mass of the Black Hole}

The precision of our measurement of the black hole mass $\left(10.91 \pm 1.55 M_{\odot}\right)$ represents a great improvement over the pioneering work of Hutchings et al. (1987) who give a mass "near $6 M_{\odot}$ " for the black hole. Prior to this work, relatively little progress was made on the dynamics of LMC X-1; for example, in their recent compilation Charles \& Coe (2006) give a range of 8-20 $M_{\odot}$ for the mass of the black hole in LMC X-1. Most of the binaries that contain dynamically confirmed black holes have low mass secondary stars (where $M_{2} \lesssim 2.5 M_{\odot}$ ), and the sample size of black hole binaries with high mass secondaries (where $M_{2} \gtrsim 10 M_{\odot}$ ) is rather limited at the moment. There are three systems with high mass secondaries where dynamical studies unequivocably show that the compact object must be a black hole: LMC X-1, M33 X-7, and Cyg $\mathrm{X}-1$. The component masses of M33 X-7 are well constrained $\left(M=15.65 \pm 1.45 M_{\odot}\right.$ and $M_{2}=70.0 \pm 6.9 M_{\odot}$; Orosz et al. 2007), whereas there is some disagreement on the parameters of Cyg X-1 (Herrero et al. 1995 give masses of $\approx 10 M_{\odot}$ and $18 M_{\odot}$ for the black hole and secondary star, respectively while Charles \& Coe give $>4.8 M_{\odot}$ for the black hole mass). There are two other eclipsing high mass X-ray binaries to mention in this context. 4U 1700-377 has a compact object with a mass of around $2.4 M_{\odot}$, but this object may be a massive neutron star (Clark et al. 2002). IC $10 \mathrm{X}-1$ has a large optical mass function that was measured using the $\mathrm{He}$ II $\lambda 4686$ emission line (7.64 $\pm 1.26 M_{\odot}$; Silverman \& Filippenko 2008; Prestwich et al. 2007). Assuming the He II line properly tracks the motion of

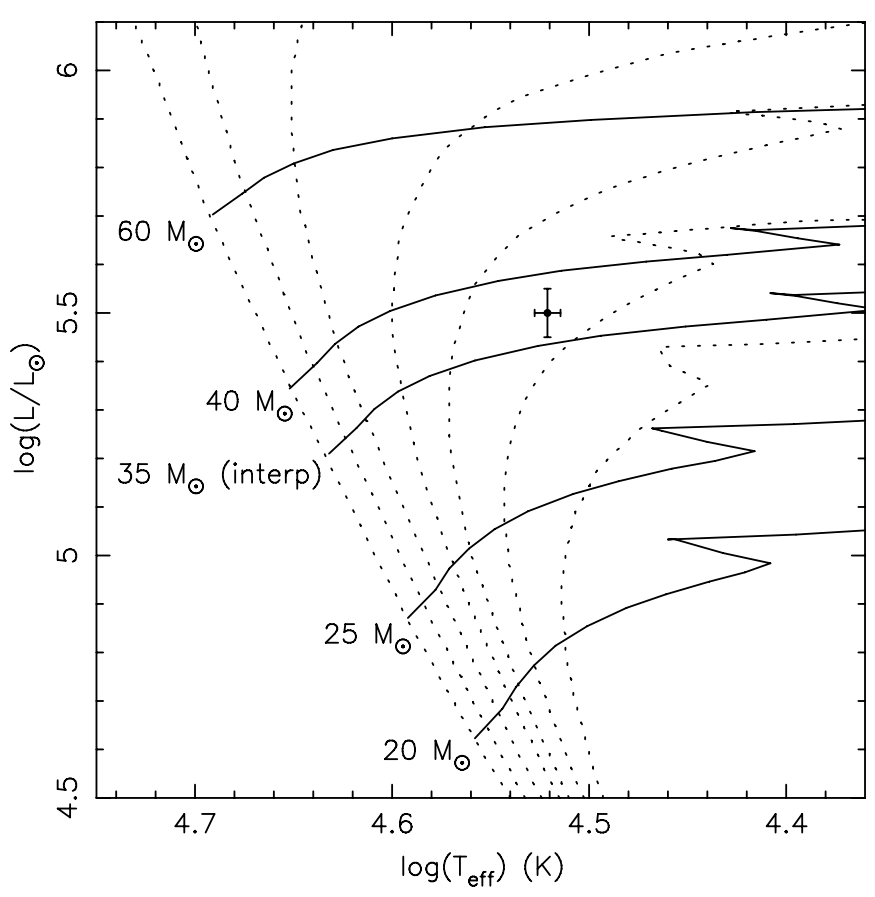

Figure 14. Solid lines show evolutionary tracks for an LMC metallicity on a temperature-luminosity diagram for stars with ZAMS masses of $60 M_{\odot}$, $40 M_{\odot}, 25 M_{\odot}, 20 M_{\odot}$ taken from Meynet et al. (1994). The track for a ZAMS mass of $35 M_{\odot}$ was crudely interpolated. The dotted lines show isochrones (also for an LMC metallicity; Lejeune \& Schaerer 2001) from left to right of 0 , $1,2,3,4,5$, and $6 \mathrm{Myr}$. The location of the LMC X-1 secondary is very close to the interpolated track for the $35 M_{\odot}$ star and the $5 \mathrm{Myr}$ isochrone.

the Wold-Rayet (WR) secondary star, and assuming a mass for the WR star based on evolutionary models, the compact object in IC $10 \mathrm{X}-1$ has a mass of $\approx 30 M_{\odot}$ or more. Fortunately, the future for studies of black hole binaries with high mass secondaries appears promising. Deep X-ray surveys of nearby galaxies are finding more and more high mass $\mathrm{X}$-ray binaries that can be followed up in ground-based studies with the new generation of large telescopes and high-performance focal-plane instrumentation.

\subsection{Evolutionary Status of the Secondary Star}

Using our measured temperature and luminosity of the secondary star $\left(33,200 \pm 500 \mathrm{~K}\right.$ and $\left.\log L / L_{\odot}=5.50 \pm 0.05\right)$ we can place the star on a temperature-luminosity diagram (Figure 14). The position of the star in the diagram can be compared with theoretical evolutionary tracks for single stars (Meynet et al. 1994) and with theoretical isochrones (Lejeune \& Schaerer 2001). Although we recognize that the evolutionary models for massive stars are uncertain owing to assumptions regarding stellar winds and rotation, for the 
purposes of this discussion we will take the evolutionary tracks and the isochrones at face value. The position of the LMC X-1 secondary is very close to the $5 \mathrm{Myr}$ isochrone. Meynet et al. (1994) give evolutionary tracks for zero-age main-sequence (ZAMS) masses of $20,25,40$, and $60 M_{\odot}$. To get a crude idea of where the evolutionary track of a star with an initial mass of $35 M_{\odot}$ would be, we plotted the initial luminosity versus the initial mass and the initial temperature versus the initial mass for the tabulated models and used quadratic interpolation to determine the initial temperature and luminosity of a star with a ZAMS mass of $35 M_{\odot}$. The evolutionary track for the star with a ZAMS mass of $40 M_{\odot}$ was then shifted down to the interpolated position of the $35 M_{\odot}$ star and plotted. The interpolated track with a ZAMS mass of $35 M_{\odot}$ passes very near the position occupied by the LMC X-1 secondary. The star with a ZAMS mass of $40 M_{\odot}$ has lost about $2.6 M_{\odot}$ by the time its temperature has fallen to $\approx 33,000 \mathrm{~K}$. If a star with a ZAMS mass of $35 M_{\odot}$ behaves in a similar manner, then its mass would be $\approx 32.4 M_{\odot}$ when its temperature has dropped to $33,000 \mathrm{~K}$, which is consistent with what we measure $\left(M_{2}=31.79 \pm 3.48 M_{\odot}\right.$, Table 3).

As one can see from the increasing space between the isochrones, as the age increases these massive stars move quickly across the temperature-luminosity diagram. Since the secondary star in LMC X-1 nearly fills its Roche lobe (the fraction filled is about $90 \%$ ), it will soon enter into an interesting stage of binary star evolution. In round numbers, the star's current radius is $17 R_{\odot}$ and the radius of its Roche lobe is $19 R_{\odot}$. From interpolation of the evolutionary track, the star with a ZAMS mass of $40 M_{\odot}$ has a radius of $17 R_{\odot}$ at an age of 4.05 Myr and a radius of $19 R_{\odot}$ at an age of $4.22 \mathrm{Myr}$. If the evolution of a star with a ZAMS mass of $35 M_{\odot}$ proceeds at a similar pace, the secondary star in LMC X-1 will encounter its Roche lobe in only a few hundred thousand years. Since the secondary star is substantially more massive than the black hole, the resulting mass transfer will be rapid and may be unstable (Podsiadlowski et al. 2003). If the mass transfer is stable and the binary survives the initial phase of rapid mass transfer, the present-day secondary may become detached again in a binary with a longer orbital period. On the other hand, if the rapid mass transfer is unstable, the orbit will rapidly shrink and the binary will enter a "common envelope" (CE) phase. The outcome of the CE phase depends on how tightly bound the envelope of the star is. If the envelope is tightly bound, then the energy liberated as the two stars move towards each other will not be enough to expel the envelope, and the two stars will merge. On the other hand, if the envelope is loosely bound, then the envelope of the star can be expelled before the cores merge, leaving behind a tight binary consisting of the present-day black hole and the core of the present-day secondary star. Although detailed computations are required to assess these scenarios, it seems that the most likely outcome of the CE phase in LMC X-1 would be a merger since the envelopes of massive stars are tightly bound, and the density gradient in a main-sequence star is not nearly as steep as it is in a giant (e.g., Podsiadlowski et al. 2003). What the merger product would look like is an open question.

\subsection{The $O$-star Wind and the ASM X-ray Light Curves}

The X-ray minimum/maximum in the ASM X-ray light curves occurs at inferior/superior conjunction of the secondary star (Figure 9), as expected if the modulation is caused by scattering or absorption in a stellar wind. A simple cosine wave gives a reasonable fit to the light curves; the respective full amplitudes for the $A, B$ and $C$ channels are $0.072 \pm 0.010$, $0.077 \pm 0.11$, and $0.038 \pm 0.029$ (Figure 9 and Table 1). Thus, the modulation is largely independent of energy, which indicates that electron scattering is the main source of opacity. We now show that a simple model of Thomson scattering in a spherically symmetric stellar wind is not only reasonably consistent with the observed X-ray modulation but also yields plausible values of the wind mass flux, accretion rate, and accretion efficiency. For this section we use the values of component masses and other system parameters in the right-hand column of Table 3.

We assume that the $\mathrm{O}$ star has a radiatively driven wind like those from other hot stars and that the wind velocity has a radial dependence that follows a "beta law," i.e., $v(r)=v_{\infty}(1-b / r)^{\beta}$ where $b$ is approximately equal to the O-star photospheric radius $R_{2}$ (e.g., Dessart \& Owocki 2005; Kudritzki \& Puls 2000 , and references therein). The terminal velocity $v_{\infty}$ can be estimated from the escape velocity at the stellar surface and the photospheric temperature (Kudritzki \& Puls 2000). The escape velocity $v_{\text {esc }}$ can be estimated in turn from the mass and radius results in Table 3 . We obtain $v_{\text {esc }} \approx 590 \pm 40 \mathrm{~km} \mathrm{~s}^{-1}$. In the calculations below, we take the values of $v_{\infty} / v_{\text {esc }}$ and of $\beta$ to be like those in the wind model presented for M33 X-7 by Orosz et al. (2007), and so we adopt the values $v_{\infty}=1400 \mathrm{~km} \mathrm{~s}^{-1} \sim$ $2.4 v_{\text {esc }}$ and $\beta=1$. This value of $v_{\infty} / v_{\text {esc }}$ is appropriate for an $\mathrm{O}$ star with photospheric temperature $T_{2} \approx 33,000 \mathrm{~K}$ (Kudritzki \& Puls 2000). The wind velocity at the radius corresponding to the location of the black hole $\left(r=a=36.5 R_{\odot}\right)$ is then $v(a) \sim 740$ $\mathrm{km} \mathrm{s}^{-1}$. Adding in quadrature the transverse velocity of the black hole through the wind, we find that the gas flows by the black hole at a velocity $V \sim 880 \mathrm{~km} \mathrm{~s}^{-1}$. Given the substantial uncertainties in both the form of the velocity law and the values of the parameters, these estimates of $v(a)$ and $V$ must each be uncertain by as much as $50 \%$.

In any spherically symmetric wind model, the scattering optical depth along the line of sight to the X-ray source at superior conjunction only differs from that at inferior conjunction by the optical depth along an additional path of length $d=2 a \sin i=43.3 R_{\odot}$ at superior conjunction. Let $N_{\mathrm{e}}$ be the electron column density along this part of the superior conjunction line of sight. Then there must be a scattering optical depth along this part of the line of sight of $N_{\mathrm{e}} \sigma_{\mathrm{T}} \approx 0.07$, where $\sigma_{\mathrm{T}}$ is the Thomson cross section. This, in turn, implies $N_{\mathrm{e}} \sim 1.1 \times$ $10^{23} \mathrm{~cm}^{-2}$. The mean electron number density along the path of length $d$ is then $n_{\mathrm{e}} \sim 3.5 \times 10^{10} \mathrm{e}^{-} \mathrm{cm}^{-3}$. In our adopted wind model, this mean density is $\sim 33 \%$ higher than the density at the black hole orbital radius (and it is also $\sim 33 \%$ lower than at the point of highest density along this section of the line of sight). The gas density at the black hole orbital radius is then $\rho(a) \sim 5.3 \times 10^{-14} \mathrm{~g} \mathrm{~cm}^{-3}$. The corresponding mass loss rate in the O-star wind is $M_{W}=4 \pi a^{2} \rho(a) v(a) \sim 5 \times 10^{-6} M_{\odot} \mathrm{yr}^{-1}$.

If the wind of the O-star in LMC X-1 is only partially ionized like the winds of ordinary $\mathrm{O}$-stars, then photoelectric absorption should be manifest via a larger degree of modulation in the lower energy band ASM light curves. Is it a reasonable assumption in the case of LMC X-1 that iron and the other metals in the wind have been fully stripped of their K-shell electrons? Some support for this idea is provided by the remarkable photoionized He III nebula that surrounds LMC X-1 (Pakull \& Angebault 1986). More directly, at every point on the path described above the ionization parameter (Tarter et al. 1969) is large, $L / n R^{2}>1000$. Furthermore, the spectrum of LMC X-1 is soft (disk blackbody temperature $k T \approx 0.9 \mathrm{keV}$ ), which makes it an effective ionizing agent. Under these conditions, studies of 
similar high mass X-ray binaries support our conclusion that the wind is close to fully ionized in the region in question (McCray et al. 1984; Vrtilek et al. 2008).

A crude estimate of the accretion rate is given by the rate at which the matter in the wind enters a cylinder of radius $r_{\mathrm{c}} \approx 2 G M / V^{2}$ centered on the black hole, where $G$ is the gravitational constant, $M$ is the black hole mass, and $V$ is the (above estimated) velocity of the wind relative to the black hole (see, e.g., Shapiro \& Teukolsky 1983). The accretion rate is then given by $\dot{M}_{\mathrm{B}-\mathrm{H}} \sim \pi r_{\text {cap }}^{2} \rho(a) V$. Using the value of $M$ in Table 3 and the values of $\rho(a)$ and $V$ estimated above, we obtain $r_{\mathrm{c}} \sim 5$ $R_{\odot}$ and $\dot{M}_{\mathrm{B}-\mathrm{H}} \sim 3 \times 10^{-8} M_{\odot} \mathrm{yr}^{-1}$ with uncertainties in each value of roughly a factor of 2 .

As noted above, we find that the isotropic bolometric X-ray luminosity of LMC X-1 is quite steady over a time span of several years and is $\approx 2.3 \times 10^{38} \mathrm{erg} \mathrm{s}^{-1}$. With $M=10.91 M_{\odot}$ and the above estimated accretion rate, the implied accretion efficiency is $\eta \sim 0.1$. This is comparable to the 0.06 efficiency of a Schwarzschild black hole or the canonical value of 0.1 that is commonly used, but, considering that this efficiency estimate is uncertain by at least a factor of 2 , this may be fortuitous.

\section{SUMMARY}

We present a new dynamical model of the high mass $\mathrm{X}$ ray binary LMC X-1 based on high quality echelle spectra, extensive optical light curves, and infrared magnitudes and colors. From our optical data we find an orbital period of $P=3.90917 \pm 0.00005$ days. We present a refined analysis of the All Sky Monitor data from RXTE and find a period of $P=3.9094 \pm 0.0008$ days, which is consistent with the optical period. A simple model of Thomson scattering in the stellar wind accounts for the $7 \%$ modulation seen in the Xray light curves. We find that the $V$-band extinction to the source is much higher than previously assumed. The $V-K$ color of the star $(1.17 \pm 0.05)$ implies $A_{V}=2.28 \pm 0.06$, whereas several determinations of the column density from Xray observations give $A_{V}>2.57 \pm 0.12$. The color excess of $E(B-V)=0.44$ together with $A_{V}=2.27$ gives $R_{V}=5.18$, which is much higher than the nominal mean value in the LMC of $R_{V}=3.1$. For the secondary star we measure a radius of $R_{2}=17.0 \pm 0.8 R_{\odot}$ and a projected rotational velocity of $V_{\text {rot }} \sin i=129.9 \pm 2.2 \mathrm{~km} \mathrm{~s}^{-1}$. Using these measured properties of the companion star to constrain the dynamical model of the light and velocity curves, we find an inclination of $i=$ $36.38 \pm 1.92$, a secondary star mass of $M_{2}=31.79 \pm 3.48 M_{\odot}$, and a black hole mass of $10.91 \pm 1.41 M_{\odot}$. The present location of the secondary star in a temperature-luminosity diagram is consistent with that of a star with an initial mass of $35 M_{\odot}$ that is $5 \mathrm{Myr}$ past the ZAMS. The star nearly fills its Roche lobe $(\approx 90 \%)$ and, owing to the rapid change in radius with time in its present evolutionary state, it will encounter its Roche lobe and begin rapid and possibly unstable mass transfer in only a few hundred thousand years.

This publication makes use of data products from the Two Micron All Sky Survey, which is a joint project of the University of Massachusetts and the Infrared Processing and Analysis Center/California Institute of Technology, funded by the National Aeronautics and Space Administration, and the National Science Foundation. C.B.D., M.M.D., and M.B. gratefully acknowledge support from the National Science Foundation grant NSF-AST 0707627. The work of J.E.M. was supported in part by NASA grant NNX08AJ55G. M.B. gratefully acknowledges support from the National Science Foundation through grant AST 04-53609 for the California State University Undergraduate Research Experiences (CSUURE) program at San Diego State University. D.S. acknowledges an STFC Advanced Fellowship as well as support through the NASA Guest Observer Program. We thank Thierry Lanz for providing the OSTAR2002 bolometric corrections computed for the $K$ band. We acknowledge helpful discussions with Bram Boroson and Tim Kallman on photoionized winds. We thank Phil Massey for helpful discussion on O-stars.

\section{APPENDIX}

\section{ACCRETION DISK CONTAMINATION IN THE OPTICAL AND INFRARED BANDS}

The optical and infrared bands of interest to us for the ellipsoidal modeling $(B, V$, and $J)$ are in the Rayleigh-Jeans part of the spectrum for the emission from both the secondary star and the disk (see below). Therefore, the flux we receive in any particular band is proportional to the product of the projected area of the radiating surface $A$ and the temperature $T$.

The radius of the secondary star is $17.0 R_{\odot}$ and its photospheric temperature is $33,200 \mathrm{~K}$. Therefore, for the radiation from the star, we have

$$
\langle A T\rangle_{\mathrm{sec}}=1.5 \times 10^{29} \mathrm{~cm}^{2} \mathrm{~K}
$$

In the case of the disk, we note that the circularization radius of the captured wind material is likely to be quite small. Nevertheless, the steady state disk will probably have a large outer radius since the gas needs to get rid of its angular momentum. An extreme possibility is that the angular momentum is removed solely by tidal torques from the companion star acting on the outer regions of the disk. From the discussion in Frank et al. (2002; Equation (5.122)), the outer radius of the disk is then approximately equal to 0.9 times the Roche lobe radius. Using a standard approximation for the Roche lobe radius (Frank et al. 2002), we find for the outer radius

$$
R_{\mathrm{out}}=0.9 \times 0.462\left(\frac{M_{\mathrm{BH}}}{M_{\mathrm{BH}}+M_{\mathrm{sec}}}\right)^{1 / 3} a=6.6 \times 10^{11} \mathrm{~cm} .
$$

We further note that most of the disk will be dominated not by local viscous dissipation, but by reprocessing of radiation emitted by the inner region of the disk. Assuming that $10 \xi_{10} \%$ of the bolometric luminosity of the disk is reprocessed (the exact fraction is uncertain), the luminosity of the reprocessed radiation from the disk is $2.3 \times 10^{37} \xi_{10} \mathrm{erg} \mathrm{s}^{-1}$. For a typical reprocessing geometry, the effective temperature of the reprocessed radiation will vary as $R^{-1 / 2}$ (as compared to the steeper $R^{-3 / 4}$ variation one expects for viscously generated flux). Let us write the disk temperature as

$$
T(R)=T_{10}\left(R / 10^{10} \mathrm{~cm}\right)^{-1 / 2}
$$

and let us assume that the reprocessing region of the disk extends from $R_{\text {in }} \sim 100 R_{S}=3.1 \times 10^{8} \mathrm{~cm}$ to $R_{\text {out }}$ (the precise value of $R_{\text {in }}$ is not important for what follows). Then we have the condition (in cgs units)

$$
2.3 \times 10^{37} \xi_{10}=\int_{3.1 \times 10^{8}}^{6.6 \times 10^{11}} 4 \pi R \sigma T_{10}^{4}\left(R / 10^{10}\right)^{-2} d R .
$$


Solving, we obtain

$$
T_{10}=8.1 \times 10^{4} \xi_{10}^{1 / 4} \mathrm{~K}
$$

The disk temperature thus varies from $4.6 \times 10^{5} \xi_{10}^{1 / 4} \mathrm{~K}$ at $R_{\text {in }}$ to $1.0 \times 10^{4} \xi_{10}^{1 / 4} \mathrm{~K}$ at $R_{\text {out }}$. Even the outermost region of the disk is hot enough that we may treat its optical/IR emission in the Rayleigh-Jeans limit.

We can now estimate the value of $\langle A T\rangle$ for the disk emission:

$$
\begin{aligned}
\langle A T\rangle_{\text {disk }} & =\int_{3.1 \times 10^{8}}^{6.6 \times 10^{11}} 2 \pi R(\cos i) T_{10}\left(R / 10^{10}\right)^{-1 / 2} d R \\
& =1.5 \times 10^{28} \xi_{10} \mathrm{~cm}^{2} \mathrm{~K} .
\end{aligned}
$$

Comparing this estimate with Equation (A1), we see that the disk will contribute about $10 \%$ of the flux in the optical and infrared. Note that the uncertain parameter $\xi_{10}$ appears only with a $1 / 4$ power, so we are not sensitive to its value. However, the result is very sensitive to the assumed outer radius of the disk. If the disk is smaller than we have assumed, the disk flux will be reduced substantially, roughly as $R_{\text {out }}^{3 / 2}$.

For a wind-fed system like LMC X-1, the disk will probably get rid of much of its angular momentum by interacting directly with the incoming material. The efficiency of this process is very difficult to estimate from first principles, so we will use the eclipsing system M33 X-7 as a guide to what the outer radius of the accretion disk might be. Orosz et al. (2007) determined an outer radius of $0.45 \pm 0.03$ times the Roche radius for M33 $\mathrm{X}-7$. If the accretion disk in LMC X-1 fills $45 \%$ of its Roche lobe then the disk contribution will be $\langle A T\rangle_{\text {disk }}=5 \times 10^{27} \xi_{10}^{1 / 4}$, which is about $3.5 \%$ of the optical/infrared flux. Even this may be an overestimate. It is quite conceivable that the disk is much smaller than $45 \%$ of the Roche lobe radius, which would make the disk contamination completely insignificant.

We have ignored reprocessing of radiation from the secondary star, but one can show that it is again not important.

\section{REFERENCES}

Avni, Y. 1976, ApJ, 209, 574

Bernstein, R., Shectman, S., Gunnels, S., Mochnacki, S., \& Athey, A. 2002, Proc. SPIE, 4841, 1694

Bianchi, L., \& Pakull, M. 1985, A\&A, 146, 242

Bildsten, L., et al. 1997, ApJS, 113, 367

Cardelli, J. A., Clayton, G. C., \& Mathis, J. S. 1989, ApJ, 345, 245

Charles, P. A., \& Coe, M. J. 2006, in Cambridge Astrophys. Ser. 39, Compact stellar X-ray sources, ed. W. Lewin \& M. van der Klis (Cambridge: Cambridge Univ. Press)

Clark, J. S., Goodwin, S. P., Crowther, P. A., Kaper, L., Fairbairn, M., Langer, N., \& Brocksopp, C. 2002, A\&A, 392, 909

Cowley, A. P., Crampton, D., \& Hutchings, A. P. 1978, AJ, 83, 1619

Cowley, A. P., Schmidtke, P. C., Anderson, A. L., \& McGrath, T. K. 1995, PASP, 107,145

Cui, W., Feng, Y. X., Shang, S. N., Bautz, M. W., Garmire, G. P., \& Schulz, N. S. 2002, ApJ, 576, 357

Dessart, L., \& Owocki, S. P. 2005, A\&A, 432, 281

Eaton, J. 2008, ApJ, 681, 562

Frank, J., King, A., \& Raine, D. J. 2002, Accretion Power in Astrophysics (3rd ed.; Cambridge: Cambridge Univ. Press)

Gierliński, M., Maciołek-Niedźwiecki, A., \& Ebisawa, K. 2001, MNRAS, 325, 1253
Haardt, F., et al. 2001, ApJS, 133, 187

Heap, S. R., Lanz, T., \& Hubeny, I. 2006, ApJ, 638, 409

Herrero, A., Kudritzki, R. P., Gabler, R., Vilchez, J. M., \& Gabler, A. 1995, A\&A, 297, 556

Hut, P. 1981, A\&A, 99, 126

Hutchings, J. B., Crampton, D., \& Cowley, A. P. 1983, ApJ, 275, L43

Hutchings, J. B., Crampton, D., Cowley, A. P., Bianchi, L., \& Thompson, I. B 1987, AJ, 94, 340

Kelson, D. D. 2003, PASP, 115, 688

Kudritzki, R.-P., \& Puls, J. 2000, ARA\&A, 38, 613

Landolt, A. U. 1992, AJ, 104, 340

Lanz, T., \& Hubeny, I. 2003, ApJS, 146, 417

Lanz, T., \& Hubeny, I. 2007, ApJS, 169, 83

Lejeune, T., \& Schaerer, D. 2001, A\&A, 366, 538

Levine, A., \& Corbet, R. 2006, ATel, \#940

Levine, A. M., Bradt, H., Cui, W., Jernigan, J. G., Morgan, E. H., Remillard, R. Shirey, R. E., \& Smith, D. A. 1996, ApJ, 469, L33

Leong, C., Kellogg, E., Gursky, H., Tannenbaum, H., \& Giacconi, R. 1971, ApJ, 170, L67

Mark, H., Price, R., Rodrigues, R., Seward, F. D., \& Swift, C. D. 1969, ApJ, 155, L143

Marshall, J. L., et al. 2008, in Ground-based and Airborne Instrumentation for Astronomy II, ed. I. S. McLean \& M. M. Casali, Proc. SPIE, 7014, 701454 701454-10

Martini, P., Persson, S. E., Murphy, D. C., Birk, C., Shectman, S. A., Gunnels, S. M., \& Koch, E. 2004, Proc. SPIE, 5492, 1653

Martins, F., \& Plez, B. 2006, A\&A, 457, 637

McCray, R., Kallman, T. R., Castor, J. I., \& Olson, G. L. 1984, ApJ, 282 245

Meynet, G., Maeder, A., Schaller, G., Schaerer, D., \& Charbonnel, C. 1994, A\&AS, 103, 97

Negueruela, I., \& Coe, M. J. 2002, A\&A, 385, 517

Nowak, M. A., Wilms, J., Heindl, W. A., Pottschmidt, K., Dove, J. B., \& Begelman, M. C. 2001, MNRAS, 320, 316

Orosz, J. A., \& Hauschildt, P. H. 2000, A\&A, 364, 265

Orosz, J. A., et al. 2002, ApJ, 568, 845

Orosz, J. A., et al. 2007, Nature, 449, 872

Pakull, M. W., \& Angebault, L. P. 1986, Nature, 322, 511

Podsiadlowski, Ph., Rappaport, S., \& Han, Z. 2003, MNRAS, 341, 385

Predehl, P., \& Schmitt, J. H. M. M. 1995, A\&A, 293, 889

Prestwich, A. H., et al. 2007, ApJ, 669, L21

Rappaport, S., Joss, P. C., \& McClintock, J. E. 1976, ApJ, 206, L103

Schlegel, E. M., Marshall, F. E., Mushotzky, R. F., Smale, A. P., Weaver, K. A., Serlemitsos, P. J., Petre, R., \& Jahoda, K. M. 1994, ApJ, 422, 243

Shapiro, S. L., \& Teukolsky, S. A. 1983, Black Holes, White Dwarfs, and Neutron Stars: The Physics of Compact Objects (New York: Wiley Interscience)

Silverman, J. M., \& Filippenko, A. V. 2008, ApJ, 678, L17

Skrutskie, M. F., et al. 2006, AJ, 131, 1163

Stetson, P. B. 1987, PASP, 99, 191

Stetson, P. B. 1990, PASP, 102, 932

Stetson, P. B. 1992a, in ASP Conf. Ser. 25, Astronomical Data Analysis Software and Systems I, ed. D. M. Worrall, C. Biemesderfer, \& J. Barnes (San Francisco, CA: ASP), 297

Stetson, P. B. 1992b, in IAU Coll. 136, Stellar Photometry: Current Techniques and Future Developments, ed. C. J. Butler \& I. Elliot (Cambridge: Cambridge Univ. Press), 291

Stetson, P. B., Davis, L. E., \& Crabtree, D. R. 1991, in ASP Conf. Ser. 8, CCDs in Astronomy, ed. G. Jacoby (San Francisco, CA: ASP), 282

Tonry, J., \& Davis, M. 1979, AJ, 84, 1511

Tarter, C. B., Tucker, W. H., \& Salpeter, E. E. 1969, ApJ, 156, 493

van der Meer, A., Kaper, L., van Kerkwijk, M. H., Heemskerk, M. H. M., \& van den Heuvel, E. P. J. 2007, A\&A, 473, 523

Vrtilek, S. D., Boroson, B. S., Hunacek, A., Gies, D., \& Bolton, C. T. 2008, ApJ 678,1248

Wade, R. A., \& Horne, K. 1988, ApJ, 324, 411

Walborn, N. 1972, AJ, 77, 312

Walborn, N. 1973, AJ, 78, 1067

Walborn, N. R., \& Fitzpatrick, E. L. 1990, PASP, 102, 379

Wilson, R. E. 1979, ApJ, 234, 1054

Wilson, R. E. 1990, ApJ, 356, 613

Wilson, R. E., \& Sofia, S. 1976, ApJ, 203, 182

Xiang, J., Zhang, S. N., \& Yao, Y. 2005, ApJ, 628, 769 